\title{
An application of the generalized Fokker-Planck equation to the dynamics of dilute polymer solutionsa)
}

\author{
Ronald S. Adler ${ }^{\text {b) }}$ \\ Department of Nuclear Engineering, The University of Michigan, Ann Arbor, Michigan 48109 \\ (Received 31 January 1978)

\begin{abstract}
The nonlinear formalism developed by Zwanzig and Mori is utilized to derive a kinetic equation for the distribution of monomer phase space coordinates and a coarse-grained momentum density. Several simplifying approximations are then introduced into the exact kinetic equation. The resulting approximate description is shown to be closely related to the starting equations of the Freed-Edwards theory. The former differs, however, due to the presence of a non-Stoke's frictional term which accounts for dissipation of monomer momentum fluctuations relative to the local velocity field of the solvent. Two applications of the approximate description are considered. A derivation of an equation for the two-time configuration space distribution function $\psi\left(\mathbf{y}, \mathbf{y}^{\prime}, t\right)$ is presented, where $\mathbf{y}$ denotes the collection of monomer position vectors. It is demonstrated that $\psi\left(\mathbf{y}, \mathbf{y}^{\prime}, t\right)$ satisfies an equation similar to the Kirkwood-Riseman equation. Nonlinear couplings of the polymer distribution function to monomer momenta and the momentum density of the solvent lead to a diffusion tensor in which hydrodynamic interactions are characterized by a coarse-grained Oseen tensor. The correlation function formulation of the intrinsic viscosity proposed by Stockmayer et al. is extended to finite wavevectors and polymer concentrations. The specific viscosity is identified as the sum of two terms involving the mechanical
\end{abstract} \\ contribution to the polymer momentum flux tensor and the diffusion current of chain segments.
}

\section{INTRODUCTION}

It is well known in polymer dynamics that one must account for the excitation of hydrodynamic modes in a solvent in order to correctly predict the transport properties of a polymer solution. ${ }^{1-7}$ Traditionally, hydrodynamic interactions have been accounted for phenomenologically within the context of the Kirkwood-Riseman and Rouse-Zimm ${ }^{4,5}$ theories. These theories treat these interactions in terms of Oseen's expression for the velocity field produced by a point source [assuming laminar flow, Eq. (4.1)].

During the past several years, considerable effort has been devoted to the derivation of dynamical equations for a polymer solution from a microscopic viewpoint..$^{9-13}$ These equations are applicable to the calculation of various transport coefficients characterizing the solution. Furthermore, such derivations should clarify the underlying assumptions in the well-known phenomenological theories of polymer dynamics. Yamakawa et al. and Curtiss et al. have derived Fokker-Planck equations for the polymer configuration space distribution function. ${ }^{9-11}$ In each of these derivations a term corresponding to a diffusion tensor is identified. However, the relationship between these diffusion tensors and those of the phenomenological theories remains unclear.

There has also been some speculation regarding the correlation function formulation of the intrinsic viscosity proposed by Stockmayer et al. ${ }^{14}$ Linear response theory predicts that the frequency dependent shear viscosity may be calculated according to the following expression:

$$
\eta(w)=\frac{\beta}{V} \int_{\delta}^{\infty} d t e^{-t w t}\langle J(t), J(0)\rangle,
$$

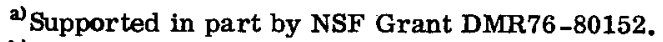

b) Present address: The James Franck Institute, The University of Chicago, Chicago, N1. 60637 .
}

where $\beta^{-1}$ is the temperature of the system (in units of energy); $V$ is the volume of the system, and the brackets refer to an equilibrium average. ${ }^{15} J(t)$ is the $x-y$ contribution to the momentum flux tensor. Starting with Eq. (1.1), Doi and Okano and Yamakawa et al. have derived correlation function formulas for the intrinsic viscosity in terms of the reduced polymer dynamics. ${ }^{9,10,16}$ Although these formulations are similar to that proposed by Stockmayer et al., hydrodynamic interactions must still be introduced phenomenologically.

The previous derivations indicate that developing a theory in terms of reduced polymer dynamics alone may be insufficient to understand the microscopic origin of the hydrodynamic interactions. On the other hand, a coupled description of the polymer-solvent dynamics, as pointed out by Freed and Edwards, automatically gives rise to interactions of the Oseen type. ${ }^{6}$

Recently, Kapral et al. have successfully incorporated hydrodynamic interactions into a microscopic calculation of the dynamic structure factor, $S(\mathbf{k}, w)$, for a polymer molecule. ${ }^{12}$ This was done using the modecoupling theory of Kawasaki and Fixman. ${ }^{17,18}$ Kapral et al. considered the bilinear coupling of the local monomer density for a chain to the coarse-grained [see Eq. (3.2)], transverse momentum density of the entire solution. Their result is in agreement with the calculation of Akcasu and Gürol. ${ }^{19}$ The latter started with the Kirkwood-Riseman equation.

The objective of this paper is the development of a dynamical theory of dilute polymer solutions from a microscopic viewpoint which takes full account of hydrodynamic excitations. The origin of these excitations is conveniently discussed in terms of the nonlinear formalism developed by Zwanzig and Mori. ${ }^{20,21} \mathrm{~A}$ brief review of the nonlinear formalism will be presented in Sec. II. In Sec. III, a kinetic equation will be derived for the collection of monomer phase space coordinates and a coarse-grained transverse momentum density. This 
choice of variables is in close analogy with that of the Freed-Edwards theory. Some simple approximations are introduced into this equation. The resulting description is then shown to have a similar structure to the starting equations of the Freed-Edwards theory (for discrete chain models). In Sec. IV, the approximate description is used to derive equations for the polymer two-time configuration space distribution function and the transverse momentum density autocorrelation function. When times much longer than collisional time scales are considered, the former reduces to a Kirkwood-Riseman-like equation. Hydrodynamic interactions are represented by a coarse-grained Oseen tensor. The frequency and wave-vector dependent specific viscosity is identified by observing the equation for the momentum density autocorrelation function. This will be written as the sum of two terms. The first of these involves only the mechanical contribution to the polymer momentum flux tensor. The second term is related to the diffusion flow of individual chain segments. A summary is presented in Sec. V.

\section{THE NONLINEAR FORMALISM}

Consider a system, in the absence of external fields, which evolves according to the laws of classical mechanics. Let

$$
\Gamma \equiv\left\{x_{1}, \ldots, p_{1}, \ldots\right\}
$$

denote the collective set of phase coordinates of the system, where $x_{i}$ and $p_{i}$ denote the position and momentum coordinates of the $i$ th particle, respectively. Furthermore, suppose that the $i$ th particle experiences a force $F_{i}$. The latter may be written in terms of a potential $U$ by the relation

$$
\mathbf{F}_{i}=-\frac{\partial}{\partial \mathbf{x}_{i}} U \text {. }
$$

Since we will be interested in the interaction of particles with a field, it will be instructive to develop the formalism using a functional notation. ${ }^{22}$ Let $\left\{A^{\alpha}(x)\right\}$ $=\left\{A^{\alpha}(x, \Gamma)\right\}$ represent a set of real fields parameterized by $x$. The time evolution of $A^{\alpha}(x)$ is determined by the classical Liouville operator

$$
A^{\alpha}(x, t)=e^{i t L} A^{\alpha}(x),
$$

where

$$
i L=\sum_{j}\left\{\frac{1}{m_{j}} \mathrm{p}_{j} \cdot \frac{\partial}{\partial \mathrm{x}_{j}}+\mathrm{F}_{j} \cdot \frac{\partial}{\partial \mathrm{p}_{j}}\right\}
$$

and $m_{j}$ is the mass of particle $j . A^{\alpha}(x)$ will be assumed to have an expansion in terms of an orthonormal basis $\left\{\varphi_{i}(x)\right\}$ :

$$
A^{\alpha}(x)=\sum_{i} A_{i}^{\alpha}(\Gamma) \varphi_{i}(x) .
$$

The set $\left\{\varphi_{i}(x)\right\}$ satisfy the usual properties of orthonormality and completeness:

$$
\begin{aligned}
& \int d x \varphi_{i}(x) \varphi_{j}(x)=\delta_{i j}, \\
& \sum_{i} \varphi_{i}(x) \varphi_{i}\left(x^{\prime}\right)=\delta\left(x-x^{\prime}\right) .
\end{aligned}
$$

Define a distribution on the space of functionals of $\left\{A^{\alpha}(x)\right\}$ by the relation

$$
S[\mathrm{a}] \equiv \prod_{\alpha} \delta\left(a^{\alpha}-A^{\alpha}\right)=\prod_{\alpha} \prod_{i} \delta\left(a_{i}^{\alpha}-A_{i}^{\alpha}\right)
$$

and

$$
\mathcal{S}[\mathbf{a} \mid t] \equiv e^{i t L} \mathrm{~S}[\mathrm{a}] .
$$

The collection $\left\{a_{1}^{\alpha}\right\}$ represent a countable infinite set of real numbers which may take on values between $\pm \infty$. Thus, the time evolution of any functional of the form $F\left[A^{1}, \ldots, A^{\alpha}, \ldots\right]=F[\mathrm{~A}]$ is completely determined by $S[\mathrm{a} \mid t]$, since

$$
F[\mathbf{A}(t)]=\int \mathcal{D}[\mathbf{a}] F[\mathbf{a}] \mathrm{S}[\mathbf{a} \mid t],
$$

where the operation

$$
\int \mathfrak{D}[\mathbf{a}] \equiv \int \prod_{\alpha} D\left[a^{\alpha}\right] \equiv \int \prod_{\alpha} \prod_{i} d a_{i}^{\alpha}
$$

denotes functional integration.

Mori has shown that $S[\mathbf{a} \mid t]$ satisfies the following equation $^{20}$ :

$$
\begin{aligned}
& \frac{\partial}{\partial t} \mathrm{~S}[\mathbf{a} \mid t]+\sum_{\alpha} \int d x \frac{\delta}{\delta a^{\alpha}(x)} v^{\alpha}[\mathbf{a} \mid x] \mathrm{S}[\mathbf{a} \mid t]-\int_{0}^{t} d \tau \sum_{\alpha, \beta} \int D\left[\mathbf{a}^{\prime}\right] \\
& \quad \times \int d x d x^{\prime} \frac{\delta}{\delta a^{\alpha}(x)} w\left[\mathbf{a}^{\prime}\right]^{-1} L^{\alpha \beta}\left[\mathbf{a}, \mathbf{a}^{\prime} \mid x, x^{\prime}, \tau\right]\left(\frac{\delta}{\delta a^{\prime \beta}\left(x^{\prime}\right)}+X^{\beta}\left[\mathbf{a}^{\prime} \mid x^{\prime}\right]\right) \mathrm{S}\left[\mathbf{a}^{\prime} \mid t-\tau\right]=-\sum_{\alpha} \int d x \frac{\delta}{\delta a^{\alpha}(x)} U(t) R^{\alpha}(x) \mathrm{S}[\mathbf{a}]
\end{aligned}
$$

or in vector notation

$\frac{\partial}{\partial t} \mathrm{~S}[\mathbf{a} \mid t]+\frac{\delta}{\delta \mathbf{a}} \cdot v[\mathbf{a}] \mathrm{S}[\mathbf{a} \mid t]-\int_{0}^{t} d \tau \int D\left[\mathbf{a}^{\prime}\right] \frac{\delta}{\delta \mathbf{a}} \cdot w\left[\mathbf{a}^{\prime}\right]^{-1} \mathrm{~L}\left[\mathbf{a}, \mathbf{a}^{\prime} \mid \tau\right] \cdot\left(\frac{\delta}{\delta \mathbf{a}^{r}}+\mathrm{X}\left[\mathbf{a}^{\prime}\right]\right) \mathrm{S}\left[\mathbf{a}^{\prime} \mid t-\tau\right]=-\frac{\delta}{\delta \mathbf{a}} \cdot U(t) \mathbf{R S}[\mathbf{a}]$.

The quantities appearing in Eq. (2.11) are defined as follows:

$$
\begin{aligned}
& w[\mathbf{a}] \equiv\langle\mathrm{S}[\mathbf{a}]\rangle, \\
& v^{\alpha}[\mathbf{a} \mid x] \equiv\left\langle i L A^{\alpha}(x), \mathrm{S}[\mathrm{a}]\right\rangle w[\mathbf{a}]^{-1},
\end{aligned}
$$

$$
\begin{aligned}
& X^{\alpha}[\mathrm{a} \mid x] \equiv-\frac{\delta}{\delta a^{\alpha}(x)} \ln w[\mathrm{a}], \\
& R^{\alpha}(x) \equiv i L A^{\alpha}(x)-\left.v^{\alpha}[\mathrm{a} \mid x]\right|_{\mathrm{a}=\mathrm{\Lambda}}, \\
& L^{\alpha \beta}\left[\mathrm{a}, \mathrm{a}^{\prime} \mid x, x^{\prime}, t\right] \equiv\left\langle U(t) R^{\alpha}(x) \mathrm{S}[\mathrm{a}], R^{\beta}\left(x^{\prime}\right) \mathrm{S}[\mathrm{a}]\right\rangle,
\end{aligned}
$$




$$
U(t) \equiv \exp \{i t(1-\odot) L\} \equiv \exp \{i t Q L\},
$$

and

$$
\mathcal{P}\{\} \equiv \int D[\mathbf{a}]\langle\{\}, S[\mathbf{a}]\rangle S[\mathbf{a}] w[\mathbf{a}]^{-1}
$$

is Zwanzig's projection operator. ${ }^{21}$ The operation $\delta / \delta a^{\alpha}(x)$ is defined by

$$
\frac{\delta}{\delta a^{\alpha}(x)} \equiv \sum_{i} \varphi_{i}(x) \frac{\partial}{\partial a_{i}^{\alpha}}
$$

and

$$
\langle F, C\rangle \equiv \int d \Gamma \rho(\Gamma) F(\Gamma) C(\Gamma)
$$

denotes an average with respect to the canonical ensemble

$$
\rho(\Gamma)=e^{-\beta x} / \int d \Gamma e^{-\beta x} .
$$

$\mathfrak{H C}$ is the system Hamiltonian.

In order to interpret the terms appearing in Eq. (2.11) it is helpful to regard the state vector $A$ as consisting of a set of local macroscopic fields (i.e., the conserved densities). $w[\mathrm{a}]$ represents the equilibrium distribution in a-space. Indeed, the equilibrium average of any functional $F[A]$ may be written as

$$
\langle F\rangle=\int d \Gamma \rho(\Gamma) F[A(\Gamma)]=\int D[\mathbf{a}] w[\mathbf{a}] F[\mathbf{a}] .
$$

The set $\left\{\mathrm{X}^{\alpha}\right\}$ may then be regarded as a set of generalized thermodynamic forces. The tensor $L\left[a, a^{\prime} \mid t\right]$ is a kinetic tensor which will be responsible for dissipation in the system. It is related to the force density RS[a] through the fluctuation dissipation theorem, Eq. (2.16). In particular, Mori referred to

$$
R^{\alpha}(x, t) \equiv U(t) R^{\alpha}(x)
$$

as the Langevin force. $\mathbf{R}(t)$ appears as the "driving force" in the nonlinear Langevin equation for $\mathbf{A}(t)$. It has the satisfying property that when averaged with respect to certain classes of constrained initial distributions (for instance, a distribution like $\exp \{-\beta[\mathcal{J C}$ $\left.\left.\left.+\int d x \alpha(x) \cdot \mathrm{A}(x)\right]\right\}\right)$ its contribution vanishes. This is in the true spirit of the Langevin equation. The vector $v[a]$ may be related to oscillations or convective flow of the local densities and, hence, represents macroscopic reversibility.

Two physical assumptions about the nature of the fields $\left\{A^{\alpha}(x)\right\}$ will be made:

(i) The time scales associated with the variation of $\left\{A^{\alpha}(x)\right\}$ are much larger than the correlation times of $\left\{R^{\alpha}(x, t)\right\}$.

(ii) A smallness is associated with the time derivatives $\dot{A}(x) \equiv i L A(x)$ (i.e., in the case of the conserved densities, smallness is associated with the magnitude of a restricted set of wave vectors).

The first of these assumptions implies the Markov nature of the variables $\left\{A^{\alpha}(x)\right\}$,

$$
L^{\alpha \beta}\left[\mathbf{a}, \mathbf{a}^{\prime} \mid x, x^{\prime}, t\right] \propto \delta(t) .
$$

The second assumption may be used as the justification to expanding the kinetic tensor, $L\left[\mathbf{a}, \mathbf{a}^{\prime} \mid t\right]$, to various orders in $\dot{A}$. Zwanzig and Mori have shown that to $O\left(\AA^{2}\right)$, L is diagonal in $a .{ }^{21,23}$ The expression given by Mori is

$$
\begin{gathered}
L^{\alpha \beta}\left[\mathbf{a}, \mathbf{a}^{\prime} \mid x, x^{\prime}, t\right]=2 \delta(t) \delta\left(\mathbf{a}-\mathbf{a}^{\prime}\right) \int_{0}^{\infty} d \tau\left\langle R^{\alpha}(x, \tau), R^{\beta}\left(x^{\prime}\right) \mathcal{G}[\mathbf{a}]\right\rangle \\
\quad+O\left(\dot{A}^{3}\right) \equiv 2 \delta(t) \delta\left(\mathbf{a}-\mathbf{a}^{\prime}\right) L^{\alpha \beta}\left[\mathbf{a} \mid x, x^{\prime}\right]+O\left(\dot{A}^{3}\right)
\end{gathered}
$$

into which the Markov assumption has also been introduced. Consistent with Eq. (2.23) one also has

$$
\begin{aligned}
-\int d x \frac{\delta}{\delta a^{\alpha}(x)} U(t) R^{\alpha}(x) \mathrm{S}[\mathbf{a}]= & -\int d x \frac{\delta}{\delta a^{\alpha}(x)} R^{\alpha}(x, t) \mathrm{G}[\mathbf{a}]+\sum_{\beta} \int d x d x^{\prime} \frac{\delta^{2}}{\delta a^{\alpha}(x) \delta a^{\beta}\left(x^{\prime}\right)} \mathrm{S}[\mathbf{a}] \\
& \times \int_{0}^{t} d \tau U(t-\tau) Q \dot{A}^{\alpha}(x) R^{\beta}\left(x^{\prime}, \tau\right)+O\left(\dot{A}^{3}\right) .
\end{aligned}
$$

Thus, Eq. (2.11) becomes

$\frac{\partial}{\partial t} \mathrm{~S}[\mathbf{a} \mid t]+\frac{\delta}{\delta \mathbf{a}} \cdot v[\mathbf{a}] \mathrm{S}[\mathbf{a} \mid t]-\frac{\delta}{\delta \mathbf{a}} \cdot w[\mathbf{a}]^{-1} \mathbf{L}[\mathbf{a}] \cdot\left(\frac{\delta}{\delta \mathbf{a}}+\mathbf{X}[\mathbf{a}]\right) \mathrm{S}[\mathbf{a} \mid t]=-\frac{\delta}{\delta \mathbf{a}} \cdot \mathbf{R}(t) \mathrm{S}[\mathbf{a}]+\frac{\delta^{2}}{\delta \mathbf{a} \delta \mathbf{a}}: \mathbf{S}[\mathbf{a}] \int_{0}^{t} d \tau U(t-\tau) Q \dot{\mathbf{A} R}(\tau)$

When one is interested in the calculation of correlation functions, it will be convenient to work with an equation for the conditional mean (defined below) of a functional of $\left\{A^{\alpha}(x)\right\}$. In order to obtain such an equation, we first define

$$
G\left[\mathbf{a}, \mathbf{a}^{\prime} \mid t\right] \equiv\left\langle\mathcal{S}[\mathbf{a} \mid t], \quad S\left[\mathbf{a}^{\prime}\right]\right\rangle / w\left[\mathbf{a}^{\prime}\right] .
$$

$G\left[a, a^{\prime} \mid t\right]$ is the conditional distribution for the state $\mathbf{A}=\mathbf{a}$ at time $t$ given the initial state $\mathbf{A}=\mathbf{a}^{\prime}$. The dynamic correlation function of two functionals, $F[A]$ and $C[A]$, is then given by

$$
\begin{aligned}
\langle F(t), C\rangle & =\int d \Gamma \rho(\Gamma) F[A(\Gamma, t)] C[A(\Gamma)] \\
& =\int \mathcal{D}[\mathbf{a}] \mathcal{D}\left[\mathbf{a}^{\prime}\right] F[\mathbf{a}] C\left[\mathbf{a}^{\prime}\right] G\left[\mathbf{a}, \mathbf{a}^{\prime} \mid t\right] w\left[\mathbf{a}^{\prime}\right] \\
& \equiv \int \mathbb{D}\left[\mathbf{a}^{\prime}\right] \bar{F}^{\mathbf{a}^{\prime}}(t) C\left[\mathbf{a}^{\prime}\right] w\left[\mathbf{a}^{\prime}\right],
\end{aligned}
$$

where the last expression serves to define the conditional mean, $\bar{F}^{\alpha^{\prime}}(t)$.

An equation for $G\left[\mathbf{a}, \mathbf{a}^{\prime} \mid t\right]$ is obtained by multiplying 
Eq. (2.25) by $\mathrm{S}\left[\mathbf{a}^{\prime}\right] w\left[\mathbf{a}^{\prime}\right]^{-1}$ and averaging the resulting equation with respect to $\rho(\Gamma)$. This results in a FokkerPlanck equation for $G\left[\mathbf{a}, \mathbf{a}^{\prime} \mid t\right]$ :

$$
\begin{aligned}
& \frac{\partial}{\partial t} G\left[\mathbf{a}, \mathbf{a}^{\prime} \mid t\right]+\frac{\delta}{\delta \mathbf{a}} \cdot v[\mathbf{a}] G\left[\mathbf{a}, \mathbf{a}^{\prime} \mid t\right] \\
& -\frac{\delta}{\delta \mathbf{a}} \cdot w[\mathbf{a}]^{-1} \mathbf{L}[\mathbf{a}] \cdot\left(\frac{\delta}{\delta \mathbf{a}}+\mathbf{X}[\mathbf{a}]\right) \\
& \quad \times G\left[\mathbf{a}, \mathbf{a}^{\prime} \mid t\right]=0 \equiv\left(\frac{\partial}{\partial t}-\Re t\right) G\left[\mathbf{a}, \mathbf{a}^{\prime} \mid t\right]=0,
\end{aligned}
$$

where it is noted that $\left\langle\mathbf{R}(t) \mathrm{S}[\mathbf{a}], G\left[\mathbf{a}^{\prime}\right]\right\rangle=0 . G\left[\mathbf{a}, \mathbf{a}^{\prime} \mid t\right]$ is the solution of this Fokker-Planck equation with the initial condition

$$
G\left[\mathbf{a}, \mathbf{a}^{\prime} \mid 0\right]=\delta\left(\mathbf{a}-\mathbf{a}^{\prime}\right) .
$$

It is also readily verified, with the aid of Eqs. (2.12)(2.14), that the equilibrium distribution, $w[\mathrm{a}]$, is a stationary solution of Eq. (2.28) as it must.

Following Mori, an operator $\Lambda$ will be defined by the relation 20,23

$$
\int \mathfrak{D}[\mathbf{a}](\mathfrak{M} F[\mathbf{a}]) C[\mathbf{a}] \equiv \int \mathscr{D}[\mathbf{a}]-F[\mathbf{a}] \Lambda C[\mathbf{a}] .
$$

The operator $\Lambda$ has the explicit form

$$
\Lambda=\boldsymbol{v}[\mathbf{a}] \cdot \frac{\delta}{\delta \mathbf{a}}+\left(\frac{\delta}{\delta \mathbf{a}}-\mathbf{X}[\mathbf{a}]\right) w[\mathbf{a}]^{-1} \cdot \tilde{\mathbf{L}}[\mathbf{a}] \cdot \frac{\delta}{\delta \mathbf{a}},
$$

where

$$
\tilde{L}^{\alpha \beta}\left[\mathbf{a} \mid x, x^{\prime}\right] \equiv L^{\beta \alpha}\left[\mathbf{a} \mid x^{\prime}, x\right]
$$

and Eq. (2.28) has been used. Applying these considerations to the definition of $\bar{F}^{a}(t)$, Eq. (2.27), yields

$$
\bar{F}^{a^{\prime}}(t)=\int D[\mathbf{a}] F[\mathbf{a}] e^{t \mathfrak{I \pi}} \delta\left(\mathbf{a}-\mathbf{a}^{\prime}\right)=e^{t \Lambda} F\left[\mathbf{a}^{\prime}\right]
$$

or

$$
\frac{\partial}{\partial t} \bar{F}^{a^{\prime}}(t)=\Lambda \bar{F}^{a^{\prime}}(t)
$$

Equation (2.32) provides a convenient starting point for the application of projection operators onto dynamical variables of interest. Similar starting points have been used in connection with the polymer problem by Bixon, Zwanzig, Akcasu, and Gürol and Freed et al. .5-27,19 $^{2}$ However, these authors have used the adjoints of the Kirkwood and Rouse (i.e., nondraining and free-draining) operators.

Although Eq. (2.32) bears a formal resemblance to original Liouville equation, there are two major advantages of having first gone to the nonlinear formalism:

(i) For a suitable choice of the set $\left\{A^{\alpha}(x)\right\}$, there is a complete separation of rapidly varying and slowly varying time scales.

(ii) The nonlinear terms, $v[\mathbf{a}]$ and $L[a]$, and the equilibrium distribution, $w[\mathbf{a}]$, may be modeled or approximated according to some physically reasonable prescription.

\section{APPLICATION TO POLYMERS}

In this section, the nonlinear formalism will be applied to a solution of identical polymer molecules satisfying

$$
n N m / c \ll 1,
$$

where $n$ is the polymer number density, $N$ is the number of segments per molecule, $m$ is the segment mass, and $c$ is the solvent mass concentration. The system will be contained in a large volume $V$.

The polymer solution will be characterized by the collective set of monomer phase space coordinates and a transverse momentum density. This set of variables will be taken as sufficient to describe the full polymersolvent dynamics. When it is necessary to distinguish between microscopic variables and their realizations, the former will include $\Gamma$ as part of their argument. Thus, microscopic position and momentum coordinates and their realizations shall be denoted by $\left\{\mathrm{x}_{i}(\Gamma), \mathrm{p}_{\mathbf{i}}(\Gamma)\right\}$ and $\left\{x_{i}, p_{i}\right\}$, respectively.

A coarse-grained, transverse momentum density will be defined by ${ }^{28}$

$\mathbf{g}_{1}(\mathbf{k}, \Gamma) \equiv \Delta(\mathbf{k}) \mathbf{S}(\mathbf{k}) \cdot \sum_{j=\text { al1 }} \sum_{\text {particles }} \mathbf{p}_{j}(\Gamma) \exp \left[i \mathbf{k} \cdot \mathbf{x}_{j}(\Gamma)\right]$,

where

$$
\begin{aligned}
& \mathbf{S}(\mathbf{k}) \equiv \mathbf{l}-\mathbf{k} \mathbf{k} k^{-2}, \\
& \Delta(\mathbf{k}) \equiv\left\{\begin{array}{ll}
1 & k \leq \pi / l \\
0 & k>\pi / l
\end{array} .\right.
\end{aligned}
$$

The length scale $l$ will be taken to be much larger than any solvent correlation length. This restriction allows the treatment of the solvent as a viscous continuum. We will also see that the presence of $\mathbf{S}(\mathbf{k})$ is equivalent to considering only incompressible modes of the solvent. The realization of $\mathbf{g}_{1}(\mathbf{k}, \Gamma)$ is $\mathbf{g}_{\perp}(\mathbf{k})$.

The dynamical vector to be considered will consist of $\left\{\mathbf{x}_{i}(\Gamma), \mathrm{p}_{i}(\Gamma)\right\}$ and $\mathbf{g}_{\mathbf{1}}(\mathbf{k}, \Gamma)$,

$$
\mathbf{A}(\Gamma) \equiv\left[\begin{array}{c}
\left\{\mathbf{x}_{i}(\Gamma) \mid i=\text { monomers }\right\} \\
\left\{\mathbf{p}_{i}(\Gamma) \mid i=\text { monomers }\right\} \\
\left\{\mathbf{g}_{\perp}(\mathbf{k}, \Gamma) \mid k \leq \pi / l\right\}
\end{array}\right] \equiv\left[\begin{array}{c}
\mathbf{x}(\Gamma) \\
\mathrm{p}(\Gamma) \\
\mathbf{g}_{\perp}(\Gamma)
\end{array}\right]
$$

and

$$
a \equiv\left[\begin{array}{c}
x \\
p \\
g_{\perp}
\end{array}\right] .
$$

The dynamical vector A, Eq. (3.5), is of a more general form than that discussed in the previous section. However, this generalization will not affect the structure of the dynamical equations for A [i.e., Eq. (2.25)]. The functional notation may be applied directly to $\mathbf{g}_{1}(\mathbf{k})$ for systems of infinite volume. However, one must keep track of the restrictions on $g_{\perp}$ and $k$. This is done most easily with the help of Eqs. (3.3)-(3.4). For example,

$$
\frac{\delta}{\delta \boldsymbol{B}_{1}(\mathbf{k})} \mathbf{g}_{1}\left(\mathbf{k}^{\prime}\right)=\Delta(\mathbf{k}) \mathbf{S}(\mathbf{k}) \delta\left(\mathbf{k}-\mathbf{k}^{\prime}\right) \text {. }
$$


Having discussed the relevant variables of the system, an equation will be obtained for

$$
\begin{aligned}
& S\left[\mathbf{x}, \mathbf{p}, \mathbf{g}_{1} \mid t\right] \equiv e^{\ddagger t \Sigma} G\left[\mathbf{x}, \mathbf{p}, \mathbf{g}_{1}\right] \\
& S\left[\mathbf{x}, \mathbf{p}, \mathbf{g}_{1}\right] \equiv \delta\left(\mathbf{g}_{1}-\mathbf{g}_{1}(\Gamma)\right) \delta(\mathbf{x}-\mathbf{x}(\Gamma)) \delta(\mathbf{p}-\mathbf{p}(\Gamma)) \\
& \quad=\prod_{k \ll r / l} \delta\left(\mathbf{g}_{1}(\mathbf{k})-\mathbf{g}_{\perp}(\mathbf{k}, \Gamma)\right) \prod_{i} \delta\left(\mathbf{x}_{i}-\mathbf{x}_{i}(\Gamma)\right) \delta\left(\mathbf{p}_{i}-\mathbf{p}_{i}(\Gamma)\right) .
\end{aligned}
$$

In terms of Eq. (3.6), Eqs. (2.12)-(2.14) become

$$
\left.\left.w\left[\mathbf{x}, \mathbf{p}, \mathbf{g}_{1}\right]=\langle S| \mathbf{x}, \mathbf{p}, \mathbf{g}_{1}\right]\right\rangle,
$$

$$
\mathrm{x}\left[\mathbf{x}, \mathbf{p}, \mathbf{g}_{1}\right]=\left[\begin{array}{c}
\mathrm{x}_{x} \\
\mathrm{x}_{\mathrm{p}} \\
\mathrm{x}_{\mathrm{r}}
\end{array}\right]=-\left[\begin{array}{c}
\left\{\frac{\partial}{\partial \mathrm{x}_{i}} \ln w\right\} \\
\left\{\frac{\partial}{\partial \mathrm{p}_{i}} \ln w\right\} \\
\left\{\frac{\delta}{\delta \mathrm{g}_{1}(\mathrm{k})} \ln w\right\}
\end{array}\right],
$$

and

$$
v\left[\mathbf{x}, \mathbf{p}, \mathbf{g}_{1}\right]=\left[\begin{array}{c}
\left\{\left\langle\dot{\mathbf{x}}_{i}, \mathcal{S}\right\rangle w^{-1}\right\} \\
\left\{\left\langle\dot{\mathbf{p}}_{i}, \mathcal{S}\right\rangle w^{-1}\right\} \\
\left\{\left\langle\dot{\mathbf{g}}_{1}(\mathbf{k}), \mathcal{S}\right\rangle w^{-1}\right\}
\end{array}\right],
$$

where $\left(^{*}\right) \equiv i L()$. Using a property of the $\delta$-function re sults in

$$
\left\langle\dot{\mathrm{x}}_{i}, S\right\rangle w^{-1}=m^{-1} \mathrm{p}_{i} \text {. }
$$

Since the momentum density $\mathrm{g}_{1}$ is a conserved variable, $\dot{\mathbf{g}}_{1}(\mathbf{k}, \Gamma) \propto k$. Define

$$
\dot{\mathbf{g}}_{1}(\mathrm{k}, \Gamma) \equiv i \mathrm{k} \cdot \Pi_{1}(\mathrm{k}) \text {. }
$$

Substituting Eqs. (3.12) and (3.13) into (3.11) yields

$$
v\left[\mathbf{x}, \mathbf{p}, \mathbf{g}_{1}\right]=\left[\begin{array}{c}
\left\{\mathbf{p}_{i} m^{-1}\right\} \\
\left\{\left\langle\dot{\mathrm{p}}_{i}, S\right\rangle w^{-1}\right\} \\
\left\{i \mathbf{k} \cdot\left\langle\Pi_{\perp}(\mathbf{k}), S\right\rangle w^{-1}\right\}
\end{array}\right] .
$$

The expression for R, Eq. (2.15), may now be evaluated:

$$
\mathbf{R}=\left[\begin{array}{c}
\{0\} \\
\left\{\dot{\mathbf{p}}_{i}(\Gamma)-\left.\left\langle\dot{\mathbf{p}}_{i}, \mathcal{S}\right\rangle w^{-1}\right|_{\mathbf{2 = \mathbf { A }}}\right\} \\
\left\{i \mathbf{k} \cdot\left(\Pi_{1}(\mathbf{k})-\left.\left\langle\Pi_{1}(\mathbf{k}), \mathcal{S}\right\rangle w^{-\mathbf{1}}\right|_{\mathbf{2 = A}}\right)\right\}
\end{array}\right] \equiv\left[\begin{array}{c}
\{0\} \\
g \\
\mathbf{R}_{k}
\end{array}\right] .
$$

Let us write $\dot{\mathrm{p}}_{i}(\Gamma)$ as the sum of two terms:

$$
\dot{\mathrm{p}}_{i}(\Gamma)=-\frac{\partial}{\partial \mathrm{X}_{i}}\left(U_{p}+U_{I}\right),
$$

where $U_{p}$ involves only monomer coordinates, and $U_{I}$ is the polymer-solvent interaction. Thus,

$$
\tilde{s}_{i}=-\frac{\partial}{\partial \mathbf{x}_{i}} U_{I}+\left.\left\langle\frac{\partial}{\partial \mathbf{x}_{i}} U_{I}, S\right\rangle w^{-1}\right|_{2=\mathbf{A}}
$$

or $\mathscr{S}_{i}$ is determined by the polymer-solvent interaction. $\mathscr{F}_{i}$ is analogous to the fluctuating force which appears in the theory of Brownian motion.

As discussed earlier, the approximate form of the kinetic tensor involves a Markov assumption and requires that $[\delta / \delta$ a.$Q \dot{A}]$ be small. ${ }^{24}$ The former relies on the assertion that collisional time scales within the solvent, and those of the solvent with monomers, are much shorter than the time scales characterizing $\left\{\mathbf{x}, \mathbf{p}, \mathbf{g}_{1}\right\}$. The latter, however, requires that we discuss the contributions of $\mathbf{x}, p$, and $\mathbf{g}_{i}$ to $L$ separately.

From Eq. (3.15), we see that the $x$ component of $R$ is zero. Therefore, only $p$ and $g_{1}$ contribute to $L$. Secondly, note that $\dot{\mathbf{g}}_{1}(\mathbf{k}, \Gamma)=i \mathbf{k} \cdot \Pi_{\perp}(\mathbf{k}) \leqslant O(1 / l)$. The only length scales entering into the calculation of $L\left[a, a^{\prime} \mid t\right]$ are solvent correlation lengths $\left\{\lambda_{s}\right\}$. Therefore, the condition $\lambda_{s} / l \ll 1$ should be sufficient to guarantee the smallness of $\dot{\mathrm{g}}_{\perp}$.

Previous theories of Brownian motion have utilized an expansion of the kinetic tensor into powers of $\epsilon=\left(m_{s} / m\right)^{1 / 2}$, where $m_{s}$ is the mass of a solvent particle. ${ }^{2 \theta}$ Yamakawa et al. point out, however, that in a polymer solution $\epsilon \sim 1 .^{9}$ In order to justify this expansion, therefore, note that each monomer is coupled to its neighbors through strong bonding potentials. Clearly, the energy associated with bonding is much larger than that associated with polymer-solvent interactions. Let $U_{I}$ [defined by Eq. (3.16)] be replaced by $\lambda U_{I}$, where $\lambda$ measures the relative strength of the polymer-solvent interaction as compared to bonding. The contribution of $p_{i}$ to the expansion for the kinetic tensor will appear as $Q \dot{p}_{i}=O(\lambda)$ which may be taken to lowest order provided that $\lambda \ll 1$.

In terms of Eqs. (2.23) and (3.15), L[a] is given by

$$
\mathbf{L}\left[\mathbf{x}, \mathbf{p}, \mathbf{g}_{1}\right]=\int_{0}^{\infty} d t\left[\begin{array}{ccc}
\{0\} & \{0\} & \{0\} \\
\{0\} & \langle U(t) \mathscr{S}, \mathscr{F S}\rangle & \left\langle U(t) \mathscr{S}, \mathbf{R}_{\boldsymbol{g}} \mathrm{S}\right\rangle \\
\{0\} & \left\langle U(t) \mathbf{R}_{e}, \mathscr{F S}\right\rangle & \left\langle U(t) \mathbf{R}_{\boldsymbol{g}}, \mathbf{R}_{\boldsymbol{g}} S\right\rangle
\end{array}\right]
$$

$$
\equiv\left[\begin{array}{ccc}
\{0\} & \{0\} & \{0\} \\
\{0\} & \left\{\mathbf{L}_{i j}\right\} & \left\{\mathbf{L}_{i g}(\mathbf{k})\right\} \\
\{0\} & \left\{\mathbf{L}_{\boldsymbol{g} i}(\mathbf{k})\right\} & \left\{\mathbf{L}_{s s}\left(\mathbf{k}, \mathbf{k}^{\prime}\right)\right\}
\end{array}\right] .
$$

Substituting Eqs. (3.8)-(3.10), (3.14)-(3.16) into Eq. $(2.25)$ results in the following equation for $\mathcal{G}\left[\mathbf{x}, \mathbf{p}, \mathbf{g}_{1} \mid t\right]$ :

$$
\begin{aligned}
\frac{\partial}{\partial t} \mathbf{S}\left[\mathbf{x}, \mathbf{p}, \mathbf{g}_{1} \mid t\right]+\left\{\sum_{i}\right. & {\left[m^{-1} \mathbf{p}_{i} \cdot \frac{\partial}{\partial \mathbf{x}_{i}}-\left\langle\dot{\mathbf{p}}_{i}, \mathrm{~S}\right\rangle w^{-1} \cdot \frac{\partial}{\partial \mathrm{p}_{i}}\right]+\int d \mathbf{k} \frac{\delta}{\delta \mathbf{g}_{1}(\mathbf{k})} \cdot\left[i \mathbf{k} \cdot\left\langle\Pi_{1}(\mathbf{k}), S\right\rangle w^{-1}\right]-\sum_{i j} \frac{\partial}{\partial \mathrm{p}_{i}} \cdot \mathbf{L}_{i j} w^{-1} \cdot\left(\frac{\partial}{\partial \mathrm{p}_{j}}+\mathrm{X}_{\mathbf{p}_{j}}\right) } \\
& -\int d \mathbf{k} \sum_{i}\left[\frac{\partial}{\partial \mathrm{p}_{i}} \cdot \mathbf{L}_{i g}(\mathbf{k}) w^{-1} \cdot\left(\frac{\delta}{\delta \mathbf{g}_{1}(\mathbf{k})}+\mathbf{X}_{g}(\mathbf{k})\right)+\frac{\delta}{\delta \mathbf{g}_{1}(\mathbf{k})} \cdot \mathbf{L}_{f i}(\mathbf{k}) w^{-1} \cdot\left(\frac{\partial}{\partial \mathrm{p}_{i}}+\mathrm{X}_{p_{i}}\right)\right]
\end{aligned}
$$




$$
\begin{aligned}
& \left.-\int d \mathbf{k} d \mathbf{k}^{\prime} \frac{\delta}{\delta \mathbf{g}_{1}(\mathbf{k})} \cdot \mathbf{L}_{8 g}\left(\mathbf{k}, \mathbf{k}^{\prime}\right) w^{-1} \cdot\left(\frac{\delta}{\delta \mathbf{g}_{1}\left(\mathbf{k}^{\prime}\right)}+\mathbf{X}_{8}\left(\mathbf{k}^{\prime}\right)\right)\right\} \mathrm{S}\left[\mathbf{x}, \mathbf{p}, \mathbf{g}_{1} \mid t\right] \\
& =-\sum_{i} \frac{\partial}{\partial \mathrm{p}_{i}} \cdot \boldsymbol{F}^{t}(t) \mathrm{S}\left[\mathbf{x}, \mathbf{p}, \mathbf{g}_{1}\right]-\int d \mathbf{k} \frac{\delta}{\delta \mathbf{g}_{1}(\mathbf{k})} \cdot \mathbf{R}_{\boldsymbol{g}}(\mathbf{k}, t) \mathrm{S}\left[\mathbf{x}, \mathbf{p}, \mathbf{g}_{1}\right]+\sum_{i, j} \frac{\partial^{2}}{\partial \mathbf{p}_{i} \partial \mathbf{p}_{j}}: S\left[\mathbf{x}, \mathbf{p}, \mathbf{g}_{1}\right] \int_{0}^{t} d \tau U(t-\tau) Q \dot{p}_{i} \mathcal{F}^{\prime}(\tau) \\
& +\sum_{i} \int d \mathbf{k} \frac{\partial}{\partial \mathrm{p}_{i}} \frac{\delta}{\delta \mathbf{g}_{\perp}(\mathbf{k})}: \mathcal{S}\left[\mathbf{x}, \mathbf{p}, \mathbf{g}_{1}\right] \int_{0}^{t} d \tau U(t-\tau) Q\left[\xi i(\tau) \dot{\mathbf{g}}_{1}(\mathbf{k})+\dot{\mathrm{p}}_{i} \mathbf{R}_{\mathrm{g}}(\mathbf{k}, \tau)\right] \\
& +\int d \mathbf{k} d \mathbf{k}^{\prime} \frac{\delta^{2}}{\delta \mathbf{g}_{1}(\mathbf{k}) \delta \mathbf{g}_{1}\left(\mathbf{k}^{\prime}\right)}: \mathcal{S}\left[\mathbf{x}, \mathbf{p}, \mathbf{g}_{1}\right] \int_{0}^{t} d \tau U(t-\tau) Q \mathbf{R}_{g}(\mathbf{k}, \tau) \dot{\mathbf{g}}_{1}\left(\mathbf{k}^{\prime}\right) \text {. }
\end{aligned}
$$

Two additional assumptions will now be introduced. We expect that the only parameters needed to describe dissipation in a polymer solution are the solvent viscosity and a friction coefficient per monomer. This situation may be achieved in Eq. (3.17) by supposing that $\mathbf{L}[\mathbf{a}] w^{-1}$ is quite insensitive to changes in $a$ and that cross effects are unimportant. Therefore, $L[\mathrm{a}] w^{-1}$ will be replaced by its average and the cross effects neglected:

$$
\begin{aligned}
\mathbf{L}\left[\mathbf{x}, \mathbf{p}, \mathbf{g}_{1}\right] w^{-1} & \simeq \int d \mathbf{x} d \mathbf{p} \mathbb{D}\left[\mathbf{g}_{1}\right] w\left[\mathbf{x}, \mathbf{p}, \mathbf{g}_{1}\right]\left(\mathbf{L} w^{-1}\right) \\
& =\int_{0}^{\infty} d t\langle\mathbf{R}(t), \mathbf{R}\rangle \\
& \simeq\left[\begin{array}{lcc}
\{0\} & \{0\} & \{0\} \\
\{0\} & \left\{\delta_{i j} \int_{0}^{\infty} d t\left\langle\mathscr{F}^{i}(t), \mathscr{F}^{j}\right\rangle\right\} & \{0\} \\
\{0\} & \{0\} & \left\{\int_{0}^{\infty} d t\left\langle\mathbf{R}_{g}(\mathbf{k}, t), \mathbf{R}_{g}\left(\mathbf{k}^{\prime}\right)\right\rangle\right\}
\end{array}\right] .
\end{aligned}
$$

From symmetry consideration one has

$$
\int_{0}^{\infty} d t\left\langle\boldsymbol{F}_{i}(t), \boldsymbol{F}_{i}\right\rangle=\beta^{-1} \xi \mathbf{I}
$$

and

$$
\int_{0}^{\infty} d t\left\langle\mathbf{R}_{g}(\mathbf{k}, t), \mathbf{R}_{\boldsymbol{g}}\left(\mathbf{k}^{\prime}\right)\right\rangle=(2 \pi)^{3} \delta\left(\mathbf{k}+\mathbf{k}^{\prime}\right) \Delta(\mathbf{k}) \mathbf{S}(\mathbf{k}) k^{2} \eta(\mathbf{k}) \beta^{-1},
$$

where $\xi$ and $\eta(\mathbf{k})$ are chosen to have dimensions of a friction coefficient and viscosity, respectively. For simplicity we shall also approximate $\eta(\mathrm{k}) \simeq \eta(0) \equiv \eta$.

Equation (3.17) will still be rather complicated due to terms like $w\left[\mathbf{x}, \mathbf{p}, \mathbf{g}_{1}\right],\left\langle\dot{p}_{i}, g\right\rangle w^{-1}$ and $\left\langle\Pi_{1}, \varsigma\right\rangle w^{-1}$. These terms will, however, be amenable to a set of simple approximations which retain the basic physics of the problem (i.e., hydrodynamic coupling).

Note that $w\left[\mathbf{x}, \mathbf{p}, \mathrm{g}_{1}\right]$ may be expressed as the product of two terms:

$w\left[\mathbf{x}, \mathbf{p}, \mathbf{g}_{1}\right]=f_{0}(\mathbf{x}, \mathbf{p}) \int d \Gamma_{\text {sol }} \rho\left(\Gamma_{\mathrm{sol}} \mid \mathbf{x}, \mathbf{p}\right) \delta\left(\mathbf{u}-\mathbf{g}_{1}\left(\Gamma_{\mathrm{sol}}\right)\right)$,

where

$$
\mathbf{u}(\mathbf{k}) \equiv \mathrm{g}_{1}(\mathrm{k})-\sum_{i} \mathrm{p}_{i} e^{i \mathbf{k} \cdot \mathbf{x}_{i}} \cdot \mathbf{s}(\mathbf{k}) \Delta(\mathrm{k})
$$

is the realization of the solvent momentum density. $\Gamma_{\text {sol }}$ refers to the collection of solvent phase coordinates, and $\rho\left(\Gamma_{\text {sol }} \mid x, p\right)$ is the distribution of the solvent conditional to a set of fixed monomer coordinates. $f_{0}(\mathbf{x}, \mathbf{p})$ is the polymer equilibrium distribution:

$$
f_{0}(\mathbf{x}, \mathbf{p}) \propto \exp \left(-\beta \sum_{i} p_{1}^{2} / 2 m\right) \exp [-\beta U(\mathbf{x})],
$$

where $U(x)$ consists of $U_{p}$ plus the average effect of the solvent on a set of fixed monomers.

Since a dilute system is being considered, the coupling of $\rho\left(\Gamma_{\text {sol }} \mid \mathbf{x}, \mathbf{p}\right)$ to monomer coordinates may be ignored to zeroth order in the polymer concentration. Thus, the conditional average in Eq. (3.21) may be replaced by an average over the pure solvent distribution, $\rho\left(\Gamma_{\mathrm{sol}}\right)$. Secondly, the restriction placed on the wave vector $\mathbf{k}$, Eq. (3.4), and the fact that $\mathrm{g}_{1}\left(\Gamma_{\mathrm{sol}}\right)$ consists of a large number of contributions allows one to approximate the remaining average by

$$
\begin{aligned}
\int d \Gamma_{\text {sol }} \rho\left(\Gamma_{\text {sol }}\right) \delta\left(\mathbf{u}-\mathbf{g}_{1}\left(\Gamma_{\text {sol }}\right)\right) \\
\simeq \exp \left(-\frac{1}{2} \frac{\beta}{c} \int \frac{d \mathbf{k}}{(2 \pi)^{3}} \mathbf{u}(\mathbf{k}) \cdot \mathbf{u}(-\mathbf{k})\right) \equiv \Phi[\mathbf{u}]
\end{aligned}
$$

as a consequence of the central limit theorem. The appropriate normalization for $\Phi$ may be absorbed into the measure $D\left[\mathbf{g}_{1}\right]$. Equation (3.24) is not surprising, since for a structureless medium one would expect the distribution of $u$ to depend only on the kinetic energy of the solvent.

The thermodynamic forces, $\mathrm{x}_{p}, \mathrm{x}_{x}, \mathrm{x}_{z}$, are computed using Eqs. (3.10), (3.21)-(3.24). As a result, one obtains

$$
\begin{aligned}
& \mathbf{X}_{x_{j}}=-\frac{\partial}{\partial \mathbf{x}_{j}} \ln w=\beta \frac{\partial}{\partial \mathbf{x}_{j}} U \equiv-\beta \mathbf{F}^{j}, \\
& \mathbf{X}_{p_{j}}=-\frac{\partial}{\partial \mathbf{p}_{j}} \ln w=\beta\left[m^{-1} \mathbf{p}_{j}-c^{-1} \mathbf{u}\left(\mathbf{x}_{j}\right)\right],
\end{aligned}
$$

and

$$
\mathbf{X}_{g}(\mathbf{k})=-\frac{\delta}{\delta \mathbf{B}_{1}(\mathbf{k})} \ln w=\frac{\beta}{(2 \pi)^{3} c} \mathbf{u}(\mathbf{k}) .
$$

The factorization, $w \simeq f_{0} \Phi$, where $\Phi$ is Gaussian, suggests a convenient method to approximate the streaming terms, $\langle\dot{\mathbf{p}}, g\rangle w^{-1}$ and $i \mathbf{k} \cdot\left\langle\Pi_{\perp}(\mathbf{k}), g\right\rangle w^{-1}$. This method involves an expansion in terms of the Hermite functional polynomials, $\left\{J_{n}^{\mu_{n}}\left[\mathrm{u} \mid k_{n}\right]\right\} .^{22}$ These polynomials satisfy the completeness relation

$$
\begin{aligned}
\Phi[\mathbf{u}]^{-1} \delta\left(\mathbf{u}-\mathbf{u}^{\prime}\right)=1 & +\sum_{n=1}^{\infty} \int d k_{n} d k_{n}^{\prime} J_{n}^{\mu_{n}}\left[\mathbf{u} \mid k_{n}\right] \\
& \times K_{n}^{\mu_{n} \nu_{n}}\left(k_{n} \mid k_{n}^{\prime}\right)^{-1} J_{n}^{\nu_{n}}\left[\mathbf{u} \mid k_{n}^{\prime}\right],
\end{aligned}
$$


where

$$
\mu_{n} \equiv\left(\mu_{1}, \ldots, \mu_{n}\right), \quad k_{n} \equiv\left(k_{1}, \ldots, k_{n}\right)
$$

and

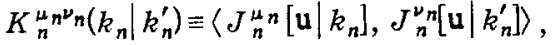

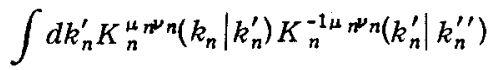

$$
\begin{aligned}
& =\prod_{i=1}^{n} \Delta\left(\mathbf{k}_{i}\right) S_{\mu_{i} \nu_{i}}\left(\mathbf{k}_{i}\right) \delta\left(\mathbf{k}_{i}-\mathbf{k}_{i}^{\prime}\right) .
\end{aligned}
$$

Expansions for the terms $\left\langle\dot{\mathbf{p}}_{i}, g\right\rangle w^{-1}$ and $i \mathbf{k} \cdot\left\langle\Pi_{1}(\mathbf{k}), g\right\rangle w^{-1}$ are obtained by replacing $\Phi[\mathrm{u}]^{-1} \delta\left(\mathrm{u}-\mathrm{g}_{1}\left(\Gamma_{\mathrm{sol}}\right)\right)$ by Eq. (3.28). ${ }^{30}$ Truncation of this expansion at some finite order is consistent with the idea that there is a weak statistical coupling of $u$ to monomer coordinates. How ever, the resulting series cannot be truncated indepen- dently of one another. One must require that each truncation preserves the stationarity of $w=f_{0} \Phi$.

The lowest order nonvanishing approximation of the streaming terms involves $J_{0}[\mathbf{u}]=1$ :

$$
\begin{aligned}
& \left\langle\dot{\mathbf{p}}_{i}, g\right\rangle w^{-1} \simeq\left\langle\dot{\mathbf{p}}_{i}, \delta(\mathbf{x}-\mathbf{x}(\Gamma)) \delta(\mathbf{p}-\mathrm{p}(\Gamma))\right\rangle f_{0}^{-1}=\mathbf{F}^{i} \\
& i \mathbf{k} \cdot\left\langle\Pi_{1}(\mathbf{k}), g\right\rangle w^{-1} \simeq i \mathbf{k} \cdot\left\langle\Pi_{\perp}(\mathbf{k}), \delta(\mathbf{x}-\mathbf{x}(\Gamma)) \delta(\mathrm{p}-\mathrm{p}(\Gamma))\right\rangle f_{0}^{-1} \\
& \quad=\sum_{i} e^{i \mathbf{k} \cdot \mathbf{x}_{i}}\left[i \mathbf{k} \cdot \mathrm{p}_{i} \mathrm{p}_{i} m^{-1}+\mathbf{F}^{i}\right] \cdot \mathbf{s}(\mathbf{k}) \Delta(\mathbf{k}),
\end{aligned}
$$

where Eqs. (3.25) and (3.13) have been used. It should be noted that this approximation is equivalent to assuming a weak dependence of the streaming terms on $u$, thereby allowing the streaming terms to be replaced by their averages with respect to $\Phi[u]$.

Applying these approximations to Eq. (3.17) results in

$$
\begin{aligned}
& \frac{\partial}{\partial t} \mathrm{~S}\left[\mathbf{x}, \mathbf{p}, \mathbf{g}_{\perp} \mid t\right]+\left\{\sum_{j}\left[m^{-1} \mathbf{p}_{j} \cdot \frac{\partial}{\partial \mathbf{x}_{j}}+\mathbf{F}^{j} \cdot \frac{\partial}{\partial \mathbf{p}_{j}}\right]+\int d \mathbf{k} \frac{\delta}{\delta \mathbf{g}_{\perp}(\mathbf{k})} \cdot \sum_{j} e^{i \mathbf{k} \cdot \mathbf{x}_{j}}\left(i \mathbf{k} \cdot \mathbf{p}_{j} \mathbf{p}_{j} m^{-\mathbf{1}}+\mathbf{F}^{j}\right)\right. \\
& \left.-\sum_{j} \frac{\partial}{\partial \mathbf{p}_{j}} \cdot \beta^{-1} \xi\left(\frac{\partial}{\partial \mathbf{p}_{j}}+\beta\left[m^{-1} \mathbf{p}_{j}-c^{-1} \mathbf{u}\left(\mathbf{x}_{j}\right)\right]\right)-\int d \mathbf{k} \frac{\delta}{\delta \mathbf{g}_{\perp}(\mathbf{k})} \cdot k^{2}(2 \pi)^{3} \frac{\eta}{\beta}\left(\frac{\delta}{\delta \mathbf{g}_{1}(-\mathbf{k})}+\frac{\beta}{(2 \pi)^{3} c} \mathbf{u}(\mathbf{k})\right)\right\} \mathrm{S}\left[\mathbf{x}, \mathbf{p}, \mathbf{g}_{\perp} \mid t\right] \\
& =-\sum_{i} \frac{\partial}{\partial \mathrm{p}_{i}} \cdot \mathscr{F}^{i}(t) \mathrm{S}\left[\mathbf{x}, \mathbf{p}, \mathbf{g}_{1}\right]-\int d \mathbf{k} \frac{\delta}{\delta \mathbf{g}_{1}(\mathbf{k})} \cdot \mathbf{R}_{\boldsymbol{g}}(\mathbf{k}, t) \mathrm{G}\left[\mathbf{x}, \mathbf{p}, \mathbf{g}_{1}\right]+\sum_{i, j} \frac{\partial^{2}}{\partial \mathrm{p}_{i} \partial \mathrm{p}_{j}}: \mathrm{S}\left[\mathbf{x}, \mathrm{p}, \mathbf{g}_{1}\right] \\
& \times \int_{0}^{t} d \tau U(t-\tau) Q \dot{\mathrm{p}}_{i} \boldsymbol{F}^{j}(\tau)+\sum_{i} \int d \mathbf{k} \frac{\partial}{\partial \mathrm{p}_{i}} \frac{\delta}{\partial \mathrm{g}_{1}(\mathbf{k})}: \mathcal{S}\left[\mathbf{x}, \mathbf{p}, \mathbf{g}_{1}\right] \int_{0}^{t} d \tau U(t-\tau) Q\left[\mathscr{F}^{i}(\tau) \dot{\mathbf{g}}_{1}(\mathbf{k})+\dot{\mathrm{p}}_{i} \mathbf{R}_{g}(\mathbf{k}, \tau)\right] \\
& +\int d \mathbf{k} d \mathbf{k}^{\prime} \frac{\delta^{2}}{\delta \mathbf{g}_{1}(\mathbf{k}) \delta \mathbf{g}_{1}\left(\mathbf{k}^{\prime}\right)}: S\left[\mathbf{x}, \mathbf{p}, \mathbf{g}_{1}\right] \int_{0}^{t} d \tau U(t-\tau) Q \mathbf{R}_{\mathbf{z}}\left(\mathbf{k}^{\prime}, \tau\right) \dot{\mathbf{g}}_{1}(\mathbf{k}),
\end{aligned}
$$

or according to the definition of $\mathfrak{M}, \mathrm{Eq} .(2.28)$,

$$
\frac{\partial}{\partial t} S\left[\mathbf{x}, \mathbf{p}, \mathbf{g}_{\perp} \mid t\right]-\mathfrak{M} S\left[\mathbf{x}, \mathbf{p}, \mathbf{g}_{\perp} \mid t\right]=\text { Random Forces } .
$$

In order to check the stationarity of $w=f_{0} \Phi$ with respect to $\Re$, it will be sufficient to look at the effect of the streaming operator alone. The action of the nonstreaming contribution to $\mathfrak{N}$ on $w$ vanishes by construction:

$$
\begin{aligned}
& \sum_{i}\left[m^{-1} \mathrm{p}_{i} \cdot \frac{\partial}{\partial \mathbf{x}_{i}}+\mathbf{F}^{i} \cdot \frac{\partial}{\partial \mathrm{p}_{i}}\right] f_{0} \Phi=-f_{0} \Phi \sum_{i}\left[m^{-1} \mathrm{p}_{i} \cdot \frac{\partial}{\partial \mathbf{x}_{i}}+\mathbf{F}^{i} \cdot \frac{\partial}{\partial \mathrm{p}_{i}}\right] \frac{1}{2} \int \frac{d \mathbf{k}}{(2 \pi)^{3}} \frac{\beta}{c} \mathbf{u}(\mathbf{k}) \cdot \mathbf{u}(-\mathbf{k}) \\
& \quad=f_{0} \Phi \sum_{i} \frac{\beta}{c} \int \frac{d \mathbf{k}}{(2 \pi)^{3}}\left[i \mathbf{k} \cdot \mathrm{p}_{i} \mathrm{p}_{i} m^{-1}+\mathbf{F}^{i}\right] e^{i \mathbf{k} \cdot \mathbf{x}_{i}} \cdot \mathbf{u}(-\mathbf{k})=-\int d \mathbf{k} \sum_{i} e^{i \mathbf{k} \cdot \mathbf{x}_{i}}\left[i \mathbf{k} \cdot \mathrm{p}_{i} \mathrm{p}_{i} m^{-1}+\mathbf{F}^{i}\right] \cdot \frac{\delta}{\delta \mathbf{g}_{1}(\mathbf{k})} f_{0} \Phi
\end{aligned}
$$

Some physical understanding of the terms appearing in Eq. (3.31) may be gained by observing the moments of this equation with respect to $x_{i}, p_{i}, u(k)$ and $g_{1}(k)$. Taking moments with respect to the first three variables results in

$$
\begin{aligned}
\frac{\partial}{\partial t} \mathbf{x}_{i}(\Gamma, t)= & m^{-1} \mathbf{p}_{i}(\Gamma, t), \\
\frac{\partial}{\partial t} \mathbf{p}_{i}(\Gamma, t)= & \mathbf{F}^{i}(\mathbf{x}(\Gamma, t))-\xi\left[m^{-1} \mathbf{p}_{i}(\Gamma, t)-c^{-1} \mathbf{u}\left(\mathbf{x}_{i}(\Gamma, t), \Gamma, t\right)\right]+F^{i}(t), \\
\frac{\partial}{\partial t} \mathbf{u}(\mathbf{k}, \Gamma, t)= & -\frac{k^{2} \eta}{c} \mathbf{u}(\mathbf{k}, \Gamma, t)+\sum_{i} \exp \left[i \mathbf{k} \cdot \mathbf{x}_{i}(\Gamma, t)\right] \Delta(\mathbf{k}) \mathbf{S}(\mathbf{k}) \xi \\
& \times\left[m^{-1} \mathbf{p}_{i}(\Gamma, t)-c^{-1} \mathbf{u}\left(\mathbf{x}_{j}(\Gamma, t), \Gamma, t\right)\right]+\mathbf{R}_{\boldsymbol{g}}(\mathbf{k}, t)-\sum_{i} \Delta(\mathbf{k}) \mathbf{S}(\mathbf{k}) \cdot S^{t}(t) e^{i \mathbf{k} \cdot \mathbf{x}_{i}} .
\end{aligned}
$$

These equations have a structure similar to the starting equations of the Freed-Edwards theory. ${ }^{B}$ However, they differ due to the presence of a non-Stokes frictional force, $\xi\left[m^{-1} p_{j}-c^{-1} \mathbf{u}\left(x_{j}\right)\right]$, and the absence of the no-slip boundary condition at the polymer-solvent interface. The latter condition would result from averaging Eqs. (3.32)(3.34) with respect to an appropriately constrained momentum distribution. The non-Stokes term would then vanish, and the average of $i(t)$ would play the role of the Lagrange multiplier which enters the Freed-Edwards theory.

Furthermore, note that only incompressible modes of the solvent are being treated since 


$$
\begin{aligned}
& i \mathbf{k} \cdot \mathbf{u}(\mathbf{k}, \Gamma, t)=0, \\
& i \mathbf{k} \cdot \mathbf{R}_{\boldsymbol{g}}(\mathbf{k}, t)=0,
\end{aligned}
$$

which follows from Eqs. (3.2), (3.3), (3.13), (3.15), and (3.22).

The $\mathbf{g}_{\perp}(\mathbf{k})$ moment of Eq. (3.31) results in

$$
\frac{\partial}{\partial t} \mathbf{g}_{1}(\mathbf{k}, \Gamma, t)=-\frac{k^{2} \eta}{c} \mathbf{u}(\mathbf{k}, \Gamma, t)+\sum_{j} \exp \left[i \mathbf{k} \cdot \mathbf{x}_{j}(\Gamma, t)\right]\left[i \mathbf{k} \cdot \mathrm{p}_{j}(\Gamma, t) \mathbf{p}_{j}(\Gamma, t) m^{-1}+\mathbf{F}^{j}(\mathbf{x}(\Gamma, t))\right] \cdot \Delta(\mathbf{k}) \mathbf{S}(\mathbf{k})+\mathbf{R}_{\mathbf{z}}(\mathbf{k}, t)
$$

indicating a clear separation between polymer and solvent contributions to the solution viscosity.

\section{RELATION TO PREVIOUS THEORIES}

In this section, the approximate Fokker-Planck description, Eq. (3.31), will be used to discuss two aspects of polymer dynamics: the Kirkwood-Riseman equation and the correlation function expression for the specific viscosity. Hydrodynamic interactions enter into these equations through the Oseen tensor:

$$
\mathbf{T}(\mathbf{x}) \equiv \frac{1}{8_{\pi} \eta|\mathbf{x}|}\left[\mathbf{I}+\frac{\mathbf{x x}}{|\mathbf{x}|^{2}}\right]
$$

Kapral et al. have shown that $\mathbf{T}$ may be accounted for in the dynamic structure factor for a chain by considering a bilinear coupling of the local monomer density to the momentum density of the solution. ${ }^{12}$ A similar type of coupling will enter into the full configurational dynamics of a chain. This is seen by noting the equivalence of the entropic force appearing in the Kirkwood-Riseman theory to a consideration of bilinear couplings of the monomer momenta and solvent fluid velocity to the polymer configuration space distribution.

The derivations to be presented are formulated in terms of correlation functions. According to the discussion in Sec. II, a convenient starting point for the calculation of correlation functions is the equation for the conditional mean,

$$
\frac{\partial}{\partial t} \bar{F}^{a}(t)=\Lambda \bar{F}^{a}(t)
$$

The operator $\Lambda$ corresponding to Eq. (3.31) is given by

$$
\begin{aligned}
\Lambda= & \sum_{j}\left[m^{-1} \mathbf{p}_{j} \cdot \frac{\partial}{\partial \mathbf{x}_{j}}+\mathbf{F}^{j} \cdot \frac{\partial}{\partial \mathbf{p}_{j}}\right]+\int d \mathbf{k} \frac{\delta}{\delta \mathbf{B}_{1}(\mathbf{k})} \cdot \sum_{j} e^{i \mathbf{k} \cdot \mathbf{x}_{j}}\left(m^{-1} \mathbf{p}_{j} \mathbf{p}_{j} \cdot i \mathbf{k}+\mathbf{F}^{j}\right) \\
& +\sum_{j}\left(\frac{\partial}{\partial \mathbf{p}_{j}}+\beta\left[m^{-1} \mathbf{p}_{j}-c^{-1} \mathbf{u}\left(\mathbf{x}_{j}\right)\right] \cdot \xi / \beta \frac{\theta}{\partial \mathbf{p}_{j}}\right)+\int d \mathbf{k}\left(\frac{\delta}{\delta \mathbf{g}_{\perp}(-\mathbf{k})}-\frac{\beta}{(2 \pi)^{3} c} \mathbf{u}(\mathbf{k})\right) \cdot k^{2} \frac{\eta(2 \pi)^{3}}{\beta} \frac{\delta}{\delta \mathbf{g}_{\perp}(\mathbf{k})},
\end{aligned}
$$

where Eqs. (2.29) and (2.30) have been used.

The configurational dynamics of polymers may be discussed in terms of the joint distribution function, $\psi(y$, $\left.\mathrm{y}^{\prime}, t\right)$, for the initial state $\mathrm{y}^{\prime}$ and the state $y$ at time $t$. $\psi$ may be expressed as the two-time correlation function of a variable

$$
h(\mathrm{y}) \equiv \delta(\mathrm{y}-\mathrm{x})
$$

according to the relation

$$
\psi\left(\mathrm{y}, \mathrm{y}^{\prime}, t\right)=\left\langle h(\mathrm{y}, t), h\left(\mathrm{y}^{\prime}\right)\right\rangle \text {. }
$$

The solution viscosity, on the other hand, may be obtained by observing the equation for the transverse momentum density autocorrelation function:

$$
\mathbf{C}(\mathbf{k}, t)=\left\langle\mathbf{g}_{1}(\mathbf{k}, t), \mathbf{g}_{1}(-\mathbf{k})\right\rangle \text {. }
$$

$\mathbf{C}(\mathbf{k}, t)$ will be shown to satisfy an equation of the form

$$
\frac{\partial}{\partial t} \mathbf{C}(\mathbf{k}, t)=-\frac{k^{2}}{c^{*}} \int_{0}^{t} d \tau \eta_{T}(\mathbf{k}, \tau) \mathbf{C}(\mathbf{k}, t-\tau)
$$

where

$$
c^{*} \equiv c+N m n
$$

The specific viscosity is then obtained from the relation

$$
[\eta(\mathbf{k}, t)]=(\eta n N m)^{-1}\left[\eta_{T}(\mathbf{k}, t)-2 \eta \delta(t)\right] .
$$

These considerations suggest that two variables of interest are $h(\mathbf{y})$ and $\mathbf{g}_{1}(\mathbf{k})$. The projection operators onto these variables are easily constructed. As a result, one finds

$$
\odot_{h}\{\}=\int d \mathbf{y}\langle\{\}, h(\mathrm{y})\rangle h(\mathrm{y}) / f(\mathrm{y})
$$

and

$$
\Phi_{\varepsilon}\{\}=\frac{\beta}{c^{*}} \int \frac{d \mathbf{k}}{(2 \pi)^{3}}\left\langle\{\}, \mathbf{g}_{1}(-\mathbf{k})\right\rangle \cdot \mathbf{g}_{1}(\mathbf{k}) .
$$

$f(y)$ is the equilibrium distribution in configuration space. 


$$
f(y)=e^{-\beta U(y)} / \int d y^{\prime} e^{-\beta U\left(y^{\prime}\right)}
$$

As discussed earlier, hydrodynamic interactions can be related to bilinear couplings of the form $\left\{\mathbf{p}_{j} h(\mathrm{y})\right\}$ and $\{\mathbf{u}(\mathbf{k}) h(\mathbf{y})\}$. However, due to the nonorthogonality of these variables to $g_{\perp}(k)$, it will be convenient to introduce

$$
g_{j}(\mathbf{y}) \equiv\left(1-P_{\xi}\right) \mathbf{p}_{j} h(\mathbf{y})=\mathbf{p}_{j} h(\mathbf{y})-\frac{m}{c^{*}} f(\mathbf{y}) \mathbf{g}_{\perp}(\mathbf{k}) e^{-i \mathbf{k} \cdot \mathbf{y}_{j}}
$$

and

$$
\begin{aligned}
\mathscr{g}_{u}(\mathbf{k}, \mathbf{y}) & \equiv\left(1-\mathscr{P}_{\boldsymbol{g}}-\odot_{p}\right) \mathbf{u}(\mathbf{k}) h(\mathbf{y}) \\
& =\mathbf{u}(\mathbf{k}) h \mathbf{y})-\frac{c}{c^{*}} f(\mathbf{y}) \mathbf{g}_{1}(\mathbf{k})-\odot_{p} \mathbf{u}(\mathbf{k}) h(\mathbf{y})
\end{aligned}
$$

$\rho_{p}$ is the projection operator onto the set $\left\{\mathscr{g}_{j}(y)\right\}$. Its form will be given below.

Let us define

$$
\begin{aligned}
\frac{m}{\beta} \phi_{j k}\left(\mathrm{y}, \mathrm{y}^{\prime}\right) & \equiv\left\langle\mathscr{J}_{j}(\mathrm{y}), \mathscr{J}_{k}\left(\mathrm{y}^{\prime}\right)\right\rangle \\
& =\frac{m}{\beta}\left[\delta_{j k} \delta\left(\mathrm{y}-\mathrm{y}^{\prime}\right) \mid f(\mathrm{y})-\frac{m}{c^{*}} f(\mathrm{y}) f\left(\mathrm{y}^{\prime}\right) \mathbf{s}\left(\mathrm{y}_{j}-\mathrm{y}_{k}^{\prime}\right)\right],
\end{aligned}
$$

where

$$
\mathbf{S}(\mathbf{x}) \equiv \int \frac{d \mathbf{k}}{(2 \pi)^{3}} e^{-i \mathbf{k} \cdot \mathbf{x}} \mathbf{S}(\mathbf{k}) \Delta(\mathbf{k})
$$

The inverse of $\phi_{j k}$ is defined by the relation

$$
\sum_{j} \int d \mathrm{y}^{\prime} \phi_{i j}\left(\mathrm{y}, \mathrm{y}^{\prime}\right) \cdot \phi_{j k}^{-1}\left(\mathrm{y}^{\prime}, \mathrm{y}^{\prime \prime}\right)=\delta_{i k} \delta\left(\mathrm{y}-\mathrm{y}^{\prime \prime}\right) !
$$

By direct substitution into (4.16), one can verify

$\phi_{j k}^{-1}\left(\mathbf{y}^{\prime}, \mathbf{y}^{\prime \prime}\right)=\left[\delta_{j k} \mid \delta\left(\mathbf{y}^{\prime}-\mathbf{y}^{\prime \prime}\right) f\left(\mathbf{y}^{\prime}\right)^{-1}+\frac{m}{c} \mathbf{s}\left(\mathbf{y}_{j}^{\prime}-\mathbf{y}_{k}^{\prime \prime}\right)\right]$.

The projection operator onto the set $\left\{\mathscr{g}_{f}(y)\right\}$ is then given by

$\mathscr{P}_{p}\{\} \equiv \frac{\beta}{m} \sum_{j k} \int d \mathrm{y} d \mathbf{y}^{\prime}\left\langle\{\}, \mathscr{g}_{j}(\mathrm{y})\right\rangle \cdot \phi_{j k}^{-1}\left(\mathrm{y}, \mathbf{y}^{\prime}\right) \cdot \mathscr{I}_{k}\left(\mathbf{y}^{\prime}\right)$.

Applying Eqs. (4.17) and $(4.18)$ to the definition of $\mathscr{I}_{u}$, Eq. (4.13), yields

$$
\mathscr{g}_{u}(\mathrm{k}, \mathrm{y})=\mathrm{u}(\mathbf{k})(h(\mathrm{y})-f(\mathbf{y})) .
$$

The corresponding projection operator onto $\mathscr{J}_{u}$ is

$\odot_{u}\{\} \equiv \frac{\beta}{c} \int \frac{d \mathbf{k}}{(2 \pi)^{3}} d \mathbf{y}\left\langle\{\}, \mathscr{L}_{u}(-\mathbf{k}, \mathbf{y})\right\rangle \cdot \mathscr{L}_{u}(\mathbf{k}, \mathbf{y}) / f(\mathbf{y})$

Equations for the joint distribution function $\psi\left(\mathrm{y}, \mathrm{y}^{\prime}, t\right)$ and $\mathbf{C}(\mathbf{k}, t)$ may be obtained from the generalized Langevin equation for the correlation matrix of

$$
\mathbf{T} \equiv\left[\begin{array}{c}
\{h(\mathbf{y})\} \\
\left\{\mathcal{g}_{\mathbf{y}}(\mathbf{y})\right\} \\
\left\{\boldsymbol{g}_{\mathbf{\prime}}(\mathbf{k}, \mathbf{y})\right\} \\
\left\{\boldsymbol{g}_{\mathbf{1}}(\mathbf{k})\right\}
\end{array}\right] .
$$

Such an equation is obtained by applying the MoriZwanzig formalism to the equation for the conditional mean of $\mathrm{T}$ (Ref. 31):

$$
\frac{\partial}{\partial t} \overline{\mathbf{T}}^{a^{\prime}}(t)=\Lambda \overline{\mathbf{T}}^{a^{\prime}}(t) \text {. }
$$

The projection operator onto $T, \mathcal{P}_{T}$, is given by

$$
\boldsymbol{P}_{T} \equiv \boldsymbol{\rho}_{p}+\boldsymbol{\rho}_{u}+\boldsymbol{\rho}_{h}+\boldsymbol{\rho}_{\mathrm{g}}
$$

where $\left\{\odot_{p}, \odot_{u}, \odot_{h}, \odot_{s}\right\}$ are given by Eqs. (4.18), (4.20), (4.9), and (4.10), respectively. Therefore, the correlation matrix satisfies

$\frac{\partial}{\partial t}\langle\mathrm{~T}(t), \mathrm{T}\rangle-\Omega \cdot\langle\mathrm{T}(t), \mathrm{T}\rangle-\int_{0}^{t} d \tau \varphi(\tau) \cdot\langle\mathrm{T}(t-\tau), \mathrm{T}\rangle=0$,

where

$$
\begin{aligned}
& \Omega \cdot T \equiv \mathscr{P}_{T} \Lambda \mathrm{T}, \\
& \varphi(t) \cdot \mathrm{T} \equiv \mathscr{P}_{T} \Lambda q(t), \\
& \mathbf{q}(t) \equiv \exp \left[t\left(1-\mathscr{\odot}_{T}\right) \Lambda\right]\left(1-\mathscr{\odot}_{T}\right) \Lambda \mathrm{T} .
\end{aligned}
$$

Equations (4.24) $-(4.26)$ are in a different form than usu. ally presented due to the nonexistence of $\langle T, T\rangle^{-1}$. The evaluation of $\Omega \cdot T, q$ and $\varphi \cdot T$ requires a knowledge of $\Lambda T$. For the purpose of obtaining $\Lambda T$, however, it will be sufficient to look at the following quantities:

$$
\begin{aligned}
& \Lambda h(\mathbf{y})=-\sum_{j} m^{-1} \frac{\partial}{\partial \mathbf{y}_{j}} \cdot \mathbf{p}, h(\mathbf{y}), \\
& \Lambda \mathbf{g}_{1}(\mathbf{k})=-\frac{k^{2} \eta}{c} \mathbf{u}(\mathbf{k})+\sum_{j} e^{i \mathbf{k} \cdot \mathbf{x}_{j}}\left[m^{-1} i \mathbf{k} \cdot \mathbf{p}_{j} \mathbf{p}_{j}+\mathbf{F}^{j}\right] \cdot \mathbf{S}(\mathbf{k}) \Delta(\mathbf{k}),
\end{aligned}
$$

$$
\begin{aligned}
& \Lambda \mathrm{p}_{j} h(\mathrm{y})=-\sum_{l} m^{-1} \frac{\partial}{\partial \mathrm{y}_{l}} \cdot \mathrm{p}_{l} \mathrm{p}_{j} h(\mathrm{y}) \\
&+\mathrm{F}^{j}(\mathrm{y}) h(\mathrm{y})-\xi\left(\frac{\mathrm{p}_{j}}{m}-\frac{1}{c} \mathrm{u}\left(\mathrm{y}_{j}\right)\right) h(\mathrm{y}), \\
& \Lambda \mathbf{u}(\mathbf{k}) h(\mathbf{y})=-\sum_{l} m^{-1} \frac{\partial}{\partial \mathbf{y}_{l}} \cdot \mathrm{p}_{l} \mathbf{u}(\mathbf{k}) h(\mathrm{y})-\frac{k^{2} \eta}{c} \mathbf{u}(\mathbf{k}) h(\mathbf{y}) \\
& \quad+\xi \sum_{l} e^{i \mathbf{k} \cdot \mathbf{y}_{l}} \mathbf{S}(\mathbf{k}) \Delta(\mathbf{k}) \cdot\left[\mathbf{p}_{l} m^{-1}-c^{-1} \mathbf{u}\left(\mathbf{y}_{l}\right)\right] h(\mathbf{y}) .
\end{aligned}
$$

The components of $\Omega \cdot T$ are calculated in Appendix $A$ with the aid of Eq. $(4.27)-(4.30)$ and the defining relations for the components of T, Eqs. $(4.3),(4.12)$, and (4.19).

In what follows, a restriction to times $t \gg m / \xi$ will be considered. The importance of the memory matrix $\varphi$ will, therefore, be determined by the characteristic time scales that are introduced into the equation for $\langle\mathbf{T}(t), \mathbf{T}\rangle$ for times $t \gg m / \xi$. It will first be necessary to consider the form of $\mathrm{q}(t)$, Eq. (4.26). Observe that the only term in Eqs. (4.27)-(4.30) which do not involve components of $\mathrm{T}$ are $-\sum_{l} m^{-1}\left(\theta / \partial y_{l}\right)$ - $\mathrm{p}_{l} \mathrm{p}_{j} h(\mathrm{y})$ and $-\sum_{l} m^{-1}\left(\partial / \partial \mathrm{y}_{l} \cdot \mathrm{p}_{t} \mathrm{u}(\mathrm{k}) h(\mathrm{y})\right)$. These terms give rise to nonvanishing contributions to $q$ : 
$\mathbf{q}(0)=\left(1-\rho_{\mathbf{T}}\right) \Lambda \mathbf{T}=\left[\begin{array}{c}\{0\} \\ \left\{-\sum_{l}\left[m^{-1} \frac{\partial}{\partial \mathbf{y}_{l}} \cdot\left(\mathbf{p}_{l} \mathbf{p}_{j}-\mathbf{l} \frac{m}{\beta} \delta_{l l}\right) h(\mathbf{y})+\frac{1}{c^{*}} f(\mathbf{y}) \mathbf{p}_{l} \cdot \frac{\partial}{\partial \mathbf{y}_{j}} \mathbf{s}\left(\mathbf{y}_{j}-\mathbf{x}_{l}\right) \cdot \mathbf{p}_{l}\right]\right\} \\ \left\{-\sum_{l} m^{-1} \frac{\partial}{\partial \mathbf{y}_{l}} \cdot \mathbf{p}_{l} \mathbf{u}(\mathbf{k}) h(\mathbf{y})\right\} \\ \left\{\sum_{l} e^{i \mathbf{k} \cdot \mathbf{x}_{l}} i \mathbf{k} \cdot \mathrm{p}_{l} \mathbf{p}_{l} m^{-1} \cdot \mathbf{s}(\mathbf{k}) \Delta(\mathbf{k})\right\}\end{array}\right.$,

where the results of Appendix A have been used. Furthermore, there are only two independent contributions to $\mathrm{q}(0),\left\{\left[\mathrm{p}_{j} \mathrm{p}_{k}-\delta_{j k} \mid(m / \beta)\right] h(\mathrm{y})\right\}$ and $\left\{\mathrm{p}_{j} \mathrm{u}(\mathbf{k}) h(\mathrm{y})\right\}$ since

$$
\mathbf{S}(\mathbf{k}) \cdot e^{i \mathbf{k} \cdot \mathbf{x}_{j}} \mathbf{p}_{f} \mathrm{p}_{j} \cdot i \mathbf{k}=\sum_{i} \delta_{j l} \mathbf{S}(\mathbf{k}) \cdot \int d \mathbf{w} e^{i \mathbf{k} \cdot \mathbf{w}_{l}}\left\{\mathbf{p}_{j} \mathbf{p}_{l}-\mathbf{l} \delta_{j l} \frac{m}{\beta}\right\} \cdot h(\mathbf{w}) i \mathbf{k}
$$

Thus, the contribution of the memory terms are determined by the evolution of $\left\{\left(p_{j} p_{k}-\delta_{j k} I(m / \beta)\right) h(y)\right\}$ and $\left\{\mathbf{u}(\mathbf{k}) \mathrm{p}_{j} h(\mathbf{y})\right\}$ with respect to the modified evolution operator $\exp \left[t\left(1-\mathscr{\rho}_{T}\right) \Lambda\right]$. One such contribution will be

$$
I=\frac{\beta}{m} \int_{0}^{\infty} \sum_{l, l} \cdot m^{-2} f(\mathbf{z})^{-1}\left\langle\exp \left[t\left(1-\boldsymbol{\Phi}_{T}\right) \Lambda\right] \frac{\partial}{\partial \mathbf{y}_{l}} \cdot\left[\mathbf{p}_{l} \mathbf{p}_{j}-\delta_{j l} \mathbf{1} \frac{m}{\beta}\right] h(\mathbf{y}), \frac{\partial}{\partial \mathbf{z}_{l}^{\prime}} \cdot\left[\mathrm{p}_{l^{\prime}} \mathbf{p}_{k}-\delta_{l^{\prime} k}, \frac{m}{\beta}\right] h(z)\right\rangle .
$$

Note that

$$
\begin{aligned}
\left(1-\mathfrak{P}_{T}\right) \Lambda\left[\mathbf{p}_{l} \mathbf{p}_{j}-\delta_{l j}, \frac{m}{\beta}\right] h(\mathbf{y})= & -\left(1-\mathscr{\Phi}_{T}\right) \sum_{r} m^{-1} \frac{\partial}{\partial \mathbf{y}_{r}} \cdot \mathbf{p}_{r} \mathbf{p}_{l} \mathbf{p}_{j} h(\mathbf{y}) \\
& -\xi\left(2 m^{-1}\left(\mathbf{p}_{l} \mathbf{p}_{j}-\delta_{l}, \frac{m}{\beta}\right)-c^{-1}\left(\mathbf{u}\left(\mathbf{y}_{l}\right) \mathbf{p}_{j}+\mathbf{p}_{l} \mathbf{u}\left(\mathbf{y}_{j}\right)\right)\right) h(\mathbf{y}) \\
& \simeq-\xi\left(2 m^{-1}\left(\mathbf{p}_{l} \mathbf{p}_{j}-\delta_{l j}, \frac{m}{\beta}\right)-c^{-1}\left(\mathbf{u}\left(\mathbf{y}_{l}\right) \mathbf{p}_{j}+\mathbf{p}_{l} \mathbf{u}\left(\mathbf{y}_{j}\right)\right)\right) h(\mathbf{y}),
\end{aligned}
$$

where Eq. (4.2) has been used. In the last equation, it may be argued that

$$
\sum_{r} m^{-1} \frac{\partial}{\partial \mathbf{y}_{r}} \cdot \mathbf{p}_{r} \mathbf{p}_{l} \mathbf{p}_{j} h(\mathbf{y}) \sim m^{-1} \Delta^{-1} \sqrt{\frac{m}{\beta}} \mathbf{p}_{l} \mathbf{p}_{j} h(\mathbf{y}),
$$

where $\Delta$ is $\sim$ bond length. ${ }^{32}$ The streaming term has been neglected on the basis that

$$
\left(\Delta^{2} \beta \xi\right)^{-1} \ll \xi / m \text {. }
$$

In other words, the collision frequency is much larger than the frequency associated with the hopping of individual chain segments. For the purpose of estimating $I$, only diagonal terms will be retained in (4.33):

$$
I \sim(2 \beta \xi)^{-1}\left[\frac{\partial}{\partial y_{k}} \frac{\partial}{\partial y_{j}}+\delta_{j k} 1 \sum_{r} \frac{\partial^{2}}{\partial y_{r}^{2}}\right] \delta(y-z) \sim\left(\beta \xi \Delta^{2}\right)^{-1}
$$

The other contributions to $\varphi$, having a similar structure to $I$, will contribute to roughly the same order. The effect of the memory matrix may, therefore, be neglected in Eq. (4.23) provided that we assume the validity of Eq. (4.34) and

$$
(\beta \xi)^{-1} \ll \eta / c \text {. }
$$

As a result, Eq. $(4,23)$ is approximated by

$$
\frac{\theta}{\partial t}\langle\mathbf{T}(t), \mathbf{T}\rangle-\Omega \cdot\langle\mathbf{T}(t), \mathbf{T}\rangle=0
$$

A second simplification that occurs is the neglect of time derivatives like $(\partial / \partial t)\left\langle\mathscr{J}_{j}(\mathbf{y}, t), \mathbf{T}\right\rangle,(\partial / \partial t)\left\langle\mathscr{L}_{u}(\mathbf{k}, \mathbf{y}, t), \mathrm{T}\right\rangle$ as compared to terms $\sim \xi / m$. These considerations lead to the following approximate description:

$$
\begin{aligned}
& \frac{\partial}{\partial t}\langle h(\mathbf{y}, t), \mathbf{T}\rangle=-\sum_{j} m^{-1} \frac{\partial}{\partial \mathbf{y}_{j}} \cdot\left\langle\mathcal{L}_{j}(\mathbf{y}, t), \mathbf{T}\right\rangle-\sum_{j} \frac{1}{c^{*}}\left\langle\mathbf{g}_{1}\left(\mathbf{y}_{j}, t\right), \mathbf{T}\right\rangle \cdot \frac{\theta}{\partial \mathbf{y}_{j}} f(\mathbf{y}),
\end{aligned}
$$

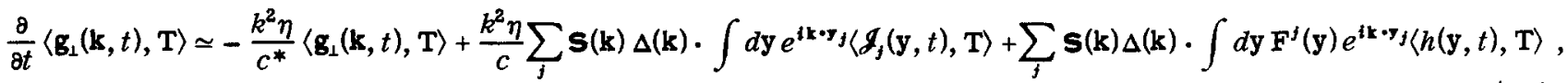

$$
\begin{aligned}
& 0 \simeq-\beta^{-1} \sum_{k} \int d \mathbf{z} \phi_{g_{k}}(\mathbf{y}, \mathbf{z}) f(\mathbf{z})^{-1} \cdot\left(\frac{\theta}{\partial \mathbf{z}_{k}}-\beta F^{k}(\mathbf{z})\right)\langle h(\mathbf{z}, t), \mathbf{T}\rangle
\end{aligned}
$$




$$
\begin{aligned}
& -\xi\left[m^{-1} \sum_{k} \int d \mathbf{z} f(\mathrm{y}) \phi_{j k}^{-1}(\mathrm{y}, \mathbf{z}) \cdot\left\langle\mathscr{g}_{k}(\mathbf{z}, t), \mathrm{T}\right\rangle-c^{-1}\left\langle\mathscr{L}_{k}\left(\mathrm{y}_{j}, \mathrm{y}, t\right), \mathrm{T}\right\rangle\right] \\
& +\frac{\eta m}{c^{*^{2}}} f(\mathbf{y}) \frac{\partial^{2}}{\partial y_{j}^{2}}\left[\frac{c^{*}}{c} \sum_{k} \int d \mathbf{z} \mathbf{S}\left(\mathbf{y}_{j}-\mathbf{z}_{k}\right) \cdot\left\langle\mathcal{g}_{k}(\mathbf{z}, t), \mathbf{T}\right\rangle-\left\langle\boldsymbol{g}_{1}\left(\mathbf{y}_{j}, t\right), \mathbf{T}\right\rangle\right] \text {, } \\
& 0 \simeq-\frac{q^{2} \eta}{c}\left\langle\mathcal{g}_{u}(\mathbf{q}, \mathbf{y}, t), \mathbf{T}\right\rangle+\xi \sum_{j} \mathbf{s}(\mathbf{q}) \Delta(\mathbf{q}) \int d \mathbf{z} e^{i \mathbf{q} \cdot \mathbf{z}_{j}}(\delta(\mathbf{y}-\mathbf{z})-f(\mathbf{y})) \\
& \times\left\{m^{-1} \sum_{k} \int d \mathbf{w} \phi_{f k}^{-1}(\mathbf{z}, \mathbf{w}) f(\mathbf{z}) \cdot\left\langle\mathcal{I}_{k}(\mathbf{w}, t), \mathbf{T}\right\rangle-c^{-1}\left\langle\mathscr{J}_{u}\left(\mathbf{z}_{j}, \mathbf{z}, t\right), \mathbf{T}\right\rangle\right\} .
\end{aligned}
$$

Equations (4.37)-(4.39) may be used to solve for $\left\langle g_{j}(y, t), T\right\rangle$. This is done in Appendix B. According to Eq. (B5), $\langle\mathcal{L}(\mathrm{y}, t), \mathrm{T}\rangle$ is given by

$$
\left\langle\mathcal{L}_{j}(\mathbf{y}, t), \mathrm{T}\right\rangle=-m \sum_{k} \int d \mathbf{z} \mathbf{D}^{j k}(\mathbf{y}, \mathbf{z}) \cdot\left(\frac{\partial}{\partial \mathbf{z}_{k}}-\beta \mathbf{F}^{k}(\mathbf{z})\right)\langle h(\mathbf{z}, t), \mathrm{T}\rangle,
$$

where

$$
\mathbf{D}^{j k}(\mathrm{y}, \mathrm{z}) \equiv \int d \mathrm{w} \sum_{l} \phi_{j l}(\mathrm{y}, \mathrm{w}) f(\mathrm{w})^{-1}\left\{\delta_{l k} \delta(\mathrm{w}-\mathrm{z})(\beta \xi)^{-1}+\mathbf{T}_{l}^{l k}(\mathbf{w}, \mathbf{z})(\delta(\mathrm{w}-\mathrm{z})-f(\mathrm{w}))\right\}
$$

and

$$
\mathbf{T}_{l}^{l k}(\mathbf{z}, \mathbf{w}) \equiv \eta^{-1} \int \frac{d \mathbf{k}}{(2 \pi)^{3}} \exp \left[-i \mathbf{k} \cdot\left(\mathbf{z}_{l}-\mathbf{w}_{k}\right)\right] k^{-2} \Delta(\mathbf{k}) \mathbf{S}(\mathbf{k})
$$

$\mathbf{T}_{l}^{l k}(\mathbf{z}, \mathbf{w})$ is a modified Oseen tensor in which the coarse-graining parameter $l$ appears explicitly. In the process of obtaining Eq. $(4.40)$, terms like $(m / \xi)(\partial / \partial t)\left\langle\mathbf{G}_{1}(\mathbf{k}, t), \mathbf{T}\right\rangle$ and $(1 / \eta l)(\mathrm{g} / \partial t)\left\langle\mathbf{B}_{1}(\mathbf{k}, t)\right.$, $\left.\mathbf{T}\right\rangle$ were ignored on the basis that only times $t \gg(m / \xi) \sim(\eta l)^{-1}$ are being considered.

We are now in a position to obtain equations for $\psi\left(\mathrm{y}, \mathrm{y}^{\prime}, t\right)$ and $\mathbf{c}(\mathbf{k}, t)$ defined by Eqs. (4.4) and (4.5). With the aid of Eqs. (4.36), (4.37), and (4.40)-(4.42) the following equation is obtained for $4\left(y, y^{\prime}, t\right)$ :

$$
\frac{\partial}{\partial t} \psi\left(\mathbf{y}, \mathbf{y}^{\prime}, t\right)=\sum_{j k} \frac{\partial}{\partial \mathrm{y}_{j}} \cdot\left(D_{l} \mid \delta_{j k}+\beta^{-1} \mathbf{T}_{l}^{j k}\left(1-\delta_{j k}\right)\right) \cdot\left(\frac{\varepsilon}{\partial \mathrm{y}_{k}}-\beta \mathrm{F}^{k}\right) \psi\left(\mathbf{y}, \mathbf{y}^{\prime}, t\right)
$$

where

$$
\begin{aligned}
& D_{l} \equiv(\beta \xi)^{-1}+(3 \pi \eta l \beta)^{-1}, \\
& \mathbf{T}_{l}^{j k} \equiv \mathbf{T}_{l}^{j k}(\mathbf{y}, \mathbf{y}) .
\end{aligned}
$$

$\mathbf{T}_{l}^{j k}$ approaches the conventional Oseen tensor, Eq. (4.1), when the limit $l-0$ is taken.

Two points are worth noting. The Kirkwood-Riseman equation follows naturally from the approximate FokkerPlanck equation, Eq. (3.31), and does not require postulating a thermodynamic force (i.e., a force proportional to the gradient of the logarithm of the distribution function). The treatment of the solvent as a structureless continuum manifests itself in the presence of a coarse-graining parameter $l$ in the hydrodynamic interaction tensor.

One may apply similar considerations in obtaining an equation for $\mathbf{c}(\mathbf{k}, t)$ :

$$
\begin{aligned}
\frac{\partial}{\partial t} \mathbf{C}(\mathbf{k}, t)= & -\frac{k^{2} \eta}{c^{*}} \mathbf{C}(\mathbf{k}, t)-\frac{\beta}{c^{*} V} \int_{0}^{t} d \tau \int d \mathbf{y} d \mathbf{z} f(\mathbf{z}) \sum_{j k l} \mathbf{S}(\mathbf{k}) \Delta(\mathbf{k}) \\
& \times e^{i \mathbf{k} \cdot \boldsymbol{\gamma}_{j}} \cdot\left[1 \delta_{f k} \delta(\mathbf{y}-\mathbf{z})-\frac{k^{2} \eta}{c} \beta \mathbf{D}^{j k}(\mathbf{y}, \mathbf{z})\right] \cdot \mathbf{F}^{k}(\mathbf{z}) \exp \left[-(t-\tau) \overline{\mathbb{S}}_{z}\right] \mathbf{F}^{l}(\mathbf{z}) e^{-i \mathbf{k} \cdot \mathbf{z}_{l}} \cdot \mathbf{C}(\mathbf{k}, t),
\end{aligned}
$$

where the operator $\overline{\mathscr{L}}_{z}$ is defined by the following relations:

$$
\begin{aligned}
& \mathscr{L}_{q} \equiv \sum_{j k} \int d w \frac{\partial}{\partial \mathbf{z}_{j}} \cdot \mathbf{D}^{j k}(\mathbf{z}, \mathbf{w}) \cdot\left(\frac{\partial}{\partial w_{k}}-\beta F^{k}(\mathbf{w})\right), \\
& f(\mathbf{z}) \overline{\mathscr{L}}_{z} \equiv \mathscr{L}_{z} f(\mathbf{z}) .
\end{aligned}
$$

Comparing Eqs. (4.45) and (4.6) enables one to identify $[\eta(\mathbf{k}, t)]$, Eq. (4.8):

$$
\begin{aligned}
& {[\eta(\mathbf{k}, t)]=(\eta n N m)^{-1} \frac{\beta}{2 k^{2} V} \mathbf{s}(\mathbf{k}): \sum_{j k} \int d \mathbf{y} f(\mathbf{y}) e^{i \mathbf{k} \cdot \mathbf{y}_{j}} \mathrm{~F}^{j}(\mathbf{y})} \\
& \times \exp \left(t \overline{\mathscr{L}}_{y}\right) e^{-i \mathbf{k} \cdot \mathbf{y}_{k}} \mathbf{F}^{k}(\mathbf{y})-(\eta n N m)^{-1} \frac{\beta \eta}{2 c V} \mathbf{s}(\mathbf{k}): \sum_{j k l} d \mathbf{y} d \mathbf{z} f(\mathbf{z}) e^{i k, y_{j}} \mathbf{D}^{j k}(\mathbf{y}, \mathbf{z}) \cdot \mathbf{F}^{k}(\mathbf{z}) \exp \left(t \overline{\mathscr{S}}_{k}\right) e^{-i \mathbf{k} \cdot \mathbf{z}_{l}} \mathbf{F}^{l}(\mathbf{z})
\end{aligned}
$$


Equation (4.49) expresses the specific viscosity as the sum of two terms. The first (f.t.) is written as an autocorrelation function of the mechanical contribution to the polymer momentum flux tensor:

$$
\sum_{j} e^{i \mathrm{r} \cdot y_{j}} \mathrm{~F}^{j}(\mathrm{y}) \text {. }
$$

The time evolution of polymer variables is determined by $\overline{\mathscr{L}}_{y}$ in which hydrodynamic interactions enter through the modified Oseen tensor, $\mathbf{T}_{l}^{t k}(\mathbf{y}, \mathbf{z})$. In the limit of infinite dilution and small $k$, f.t. approaches the form postulated by Stockmayer et al..$^{14,33}$

Physically, f.t. represents that part of the viscosity which is due to mechanical forces acting on individual chain segments. The second term (s.t.) is of a very different nature. For the purpose of understanding the presence of this term, consider an infinitely dilute system. Let us introduce the following notation:

$$
\begin{aligned}
& \psi(\mathrm{y}, t)=\text { one chain distribution function, } \\
& \mathrm{V}_{i}(\mathrm{y}, t)=\text { average velocity of monomer } i, \\
& \overline{\mathbf{u}}(\mathbf{k}, t)=\text { average solvent momentum density with } \\
& \text { wave-vector } \mathbf{k}, \\
& \overline{\mathbf{B}}_{\mathbf{i}}(\mathbf{k}, t)=\text { average momentum density of the solution. }
\end{aligned}
$$

Recall that the solvent contribution to the equation for $\overline{\mathbf{B}}_{1}(\mathbf{k}, t)[$ i.e., Eq. (3.37)] enters through a term like $\left(k^{2} \eta / c\right) \overline{\mathbf{u}}(\mathbf{k}, t) . \quad \overline{\mathbf{u}}(\mathbf{k}, t)$ may be expressed in terms of $\overline{\mathbf{B}}_{1}$ and the polymer contribution to the momentum density: $\overline{\mathbf{u}}(\mathbf{k}, t)=\overline{\mathbf{g}}_{\perp}(\mathbf{k}, t)-m n V \sum_{i=1}^{N} \int d \mathbf{y} \mathbf{S}(\mathbf{k}) \Delta(\mathbf{k}) e^{i \mathbf{k} \cdot y_{i}} \cdot \mathrm{V}_{i}(\mathrm{y}, t) \psi(\mathrm{y}, t)$.

Equation (4.50) is obtained from the following considerations:

(i) $\sum_{i=1}^{N} \mathbf{S}(\mathbf{k}) \Delta(\mathbf{k}) \cdot m e^{i \mathbf{k} \cdot y_{l}} \mathrm{~V}_{t}(\mathrm{y}, t)=$ transverse and coarse-grained momentum density for one chain given the configuration $\mathbf{y}$ at time $t$.

(ii) Now average (i) with respect to $\psi(\mathbf{y}, t)$ and multiply the result by the total number of polymers, $n V$.

The diffusion approximation corresponds to setting

$$
\mathbf{V}_{i}(\mathbf{y}, t) \psi(\mathbf{y}, t) \simeq-\sum_{j=1}^{N} \mathbf{D}^{i j} \cdot\left(\frac{\partial}{\partial \mathbf{y}_{j}}-\beta \mathbf{F}^{j}\right) \psi(\mathbf{y}, t),
$$

where, according to Eqs. (4.43)-(4.45),

$$
\mathbf{D}^{i j}=D_{l} \mathbf{i} \delta_{i j}+\left(1-\delta_{i j}\right) \mathbf{T}_{i}^{i j} \text {. }
$$

Substituting Eq. $(4.52)$ in $(4.51)$ and noting $\left(\partial / \partial y_{i}\right) \cdot D^{i j}=0$ yields

$$
\begin{gathered}
\frac{\eta k^{2}}{c} \mathbb{\mathrm { u }}(\mathbf{k}, t) \simeq \\
\frac{\eta k^{2}}{c} \overline{\mathbf{g}}_{\perp}(\mathbf{k}, t)-\frac{\eta k^{2}}{c}(\beta n m V) \sum_{i j=1}^{N} \mathbf{s}(\mathbf{k}) \Delta(\mathbf{k}) \\
\cdot \int d \mathbf{y} e^{i \mathbf{r} \cdot \mathbf{y}_{i}} \mathbf{D}^{i j} \cdot E^{j} \psi(\mathrm{y}, t)
\end{gathered}
$$

A term similar to Eq. (4.52) appears in Eq. (4,46). Thus, we see that $s . t$. arises by accounting for the diffusion flow of chain segments in the equation for the momentum density.

Finally, it should be noted that in the zero $k$ limit, s.t. vanishes. This follows from the fact that

$$
\sum_{j} e^{i \mathbf{k} \cdot y_{j}} \mathrm{~F}^{j} \sim k
$$

for small values of $k$ and, therefore, s.t. $\sim k^{2}$.

\section{SUMMARY}

This work has primarily dealt with the development of dynamical equations for a polymer solution from a mi croscopic point of view. The nonlinear formalism developed by Zwanzig and Mori has been used to derive a Fokker-Planck equation for the distribution of monomer phase space coordinates and the coarse-grained transverse momentum density, $S\left[x, p, g_{\perp} \mid t\right]$. Several simplifying approximations are introduced into the equation for $\mathrm{S}\left[\mathrm{x}, \mathrm{p}, \mathrm{g}_{\perp} \mid t\right]$. The resulting approximate description is somewhat analogous to the starting equations of the Freed-Edwards theory. There is, however, one important difference due to the presence of a non-Stokes frictional force. The former occurs when monomers are allowed to have velocity fluctuations relative to the solvent fluid velocity. Therefore, the present theory allows for slip, whereas the Freed-Edwards theory employs a no-slip boundary condition at the polymer-solvent interface.

The approximate Fokker-Planck description was then used to discuss the Kirkwood-Riseman equation and the correlation function formulation of the specific viscosity. This involved obtaining equations for the two-time configuration space distribution function, $\psi\left(\mathbf{y}, \mathbf{y}^{\prime}, t\right)$, and the momentum density autocorrelation function, $\mathbf{c}(\mathbf{k}, t)$. The former was seen to obey an equation similar to the Kirkwood-Riseman equation in which hydrodynamic interactions are present in the form of a coarse-grained Oseen tensor, $\mathbf{T}_{i}^{j k}$. The coarse-graining parameter $l$ appears as a minimum length over which the solvent may be treated as a structureless continuum. It was demonstrated that $T_{3}^{j k}$ is related to nonlinear couplings of the polymer distribution function to the monomer momenta and the coarse-grained, transverse momentum density.

The equation for $\mathbf{c}(\mathbf{k}, t)$ enabled one to identify the specific viscosity. This was expressed as the sum of two terms. The first term involved an autocorrelation function of the polymer momentum flux tensor. In the limit of infinite dilution and small wave vectors, this te rm reduces to the correlation function expression for the intrinsic viscosity proposed by Stockmayer et al. The second term could be related to the diffusion flow of chain segments. Its effect vanishes in the small $k$ limit. It should be noted that the operator governing the time evolution of polymer variables in the expression for the specific viscosity approaches the Kirkwood-Riseman operator only in the limit of infinite dilution.

\section{ACKNOWLEDGMENTS}

The work was supported by the National Science Foundation and by funds provided by a Rockham Fellowship. The author would like to express his appreciation to Professor A. Z, Akcasu, Professor G. C. Summerfield, and Professor R. Ullman, and to Professor H. GUrol and Mr. H. Matsumora for many stimulating discussions and valuable criticism of this work. 


\section{APPENDIX A: EVALUATION OF $\boldsymbol{\Omega} \cdot \boldsymbol{T}$}

The first component of $\Omega \cdot T$ is $\odot_{T} \Lambda h(y)$. This is easily evaluated by recalling the expression for $g_{j}(y)$, Eq. (4.12):

$$
\mathscr{g}_{j}(\mathrm{y})=\mathrm{p}_{j} h(\mathrm{y})-\frac{m}{c^{*}} f(\mathrm{y}) \mathrm{g}_{1}\left(\mathrm{y}_{j}\right)
$$

Therefore,

$$
\odot_{T} \Lambda h(y)=-\odot_{T} \sum_{j} \frac{\partial}{\partial y_{j}} \cdot p_{j} m^{-1} h(y)=-\sum_{j} m^{-1} \frac{\partial}{\partial y_{j}} \cdot g_{j}(y)-\frac{1}{c^{*}} \sum_{j} \cdot g_{1}\left(y_{j}\right) \cdot \frac{\partial}{\partial y_{j}} f(y) .
$$

Now consider

$$
\begin{aligned}
& \boldsymbol{F}_{\boldsymbol{T}} \Lambda \mathbf{g}_{\perp}(\mathbf{k})=\int d \mathbf{y}\left\langle\Lambda \mathbf{g}_{\perp}(\mathbf{k}), h(\mathbf{y})\right\rangle f(\mathbf{y})^{-1} h(\mathbf{y})+\frac{\beta}{c^{*}} \int \frac{d \mathbf{k}^{\prime}}{(2 \pi)^{3}}\left\langle\Lambda \mathbf{G}_{\perp}(\mathbf{k}), \mathbf{g}_{\perp}\left(-\mathbf{k}^{\prime}\right)\right\rangle \cdot \mathbf{g}_{\perp}\left(\mathbf{k}^{\prime}\right) \\
& +\frac{\beta}{m} \sum_{j \cdot k} \int d \mathbf{y} d \mathbf{y}^{\prime}\left\langle\Lambda \mathbf{g}_{\perp}(\mathbf{k}), \mathscr{L}_{j}(\mathbf{y})\right\rangle \cdot \phi_{j k}^{-1}\left(\mathbf{y}, \mathbf{y}^{\prime}\right) \cdot \mathscr{I}_{k}\left(\mathbf{y}^{\prime}\right)+\frac{\beta}{c} \int \frac{d \mathbf{k}^{\prime}}{(2 \pi)^{3}} d \mathbf{y}\left\langle\Lambda \mathbf{B}_{\perp}(\mathbf{k}), \mathcal{I}_{k}\left(-\mathbf{k}^{\prime}, \mathbf{y}\right)\right\rangle \cdot f(\mathbf{y})^{-1} \mathscr{g}_{u}\left(\mathbf{k}^{\prime}, \mathbf{y}\right) .
\end{aligned}
$$

The last term in (A2) vanishes. Note that

$$
\begin{aligned}
& \left\langle\Lambda \mathbf{g}_{\perp}(\mathbf{k}), h(\mathbf{y})\right\rangle=\sum_{j} \mathbf{s}(\mathbf{k}) \Delta(\mathbf{k}) \mathrm{F}^{j}(\mathbf{y}) e^{i \mathbf{k} \cdot \mathbf{g}_{j}} f(\mathbf{y}), \\
& \left\langle\Lambda \mathbf{g}_{1}(\mathbf{k}), \mathbf{g}_{1}\left(-\mathbf{k}^{\prime}\right)\right\rangle=-\frac{k^{2} \eta}{c}\left(\frac{c}{\beta}\right)(2 \pi)^{3} \delta\left(\mathbf{k}-\mathbf{k}^{\prime}\right) \Delta(\mathbf{k}) \mathbf{s}(\mathbf{k}), \\
& \left\langle\Lambda \mathbf{g}_{1}(\mathbf{k}), g_{j}(\mathbf{y})\right\rangle=-\frac{k^{2} \eta}{c}\left\langle\mathbf{u}(\mathbf{k}), \mathcal{g}_{j}(\mathbf{y})\right\rangle=\frac{k^{2} \eta}{c^{*}} \frac{m}{\beta} e^{i \mathbf{k} \cdot \mathbf{y}_{j}} f(\mathbf{y}) \mathbf{s}(\mathbf{k}) \Delta(\mathbf{k}) .
\end{aligned}
$$

Substitution of (A3)-(A5) into (A2) yields

$$
\mathcal{P}_{T} \Lambda \mathbf{g}_{\perp}(\mathbf{k})=-\frac{k^{2} \eta}{c} \mathbf{g}_{\perp}(\mathbf{k})+\frac{k^{2} \eta}{c} \sum_{j} \mathbf{s}(\mathbf{k}) \Delta(\mathbf{k}) \cdot \int d \mathbf{y} e^{i \mathbf{k} \cdot \mathbf{y}_{j}} g_{j}(\mathbf{y})+\sum_{j} \mathbf{s}(\mathbf{k}) \Delta(\mathbf{k}) \cdot \int d \mathbf{y} \mathbf{F}^{j}(\mathbf{y}) e^{i \mathbf{k} \cdot \mathbf{y}_{j}} h(\mathbf{y}) .
$$

The $\rho_{T} \Lambda \mathcal{I}_{\text {f }}$ contribution is given as

$$
\begin{aligned}
\mathfrak{P}_{T} \Lambda \mathscr{I}_{j}(\mathbf{y})= & \int d \mathbf{z}\left\langle\Lambda \mathscr{g}_{j}(\mathbf{y}), h(\mathbf{z})\right\rangle f(\mathbf{z})^{-1} h(\mathbf{z})+\frac{\beta}{c^{*}} \int \frac{d \mathbf{k}}{(2 \pi)^{3}}\left\langle\Lambda \mathscr{g}_{j}(\mathbf{y}), \mathbf{g}_{1}(-\mathbf{k})\right\rangle \cdot \mathbf{g}_{1}(\mathbf{k}) \\
& +\frac{\beta}{m} \sum_{l, k} \int d \mathbf{z} d \mathbf{z}^{\prime}\left\langle\Lambda \mathscr{I}_{j}(\mathbf{y}), \mathscr{I}_{l}(\mathbf{z})\right\rangle \cdot \phi_{l k}^{-1}\left(\mathbf{z}, \mathbf{z}^{\prime}\right) \cdot \mathscr{I}_{k}\left(\mathbf{z}^{\prime}\right)+\frac{\beta}{c} \int \frac{d \mathbf{k}}{(2 \pi)^{3}} d \mathbf{z}\left\langle\Lambda \mathscr{I}_{j}(\mathbf{y}), \mathscr{I}_{u}(-\mathbf{k}, \mathbf{z})\right\rangle \cdot f(\mathbf{z})^{-1} \mathscr{g}_{u}(\mathbf{k}, \mathbf{z}) .
\end{aligned}
$$

The static correlation functions in (A7) are given as follows:

$$
\left\langle\Lambda g_{j}(\mathrm{y}), h(\mathbf{z})\right\rangle=\sum_{k} m^{-1} \frac{\partial}{\partial \mathbf{z}_{k}}\left\langle\mathrm{p}_{k} h(\mathbf{z}), \mathscr{g}_{j}(\mathrm{y})\right\rangle=\beta^{-1} \sum_{k} \frac{\theta}{\partial \mathbf{z}_{k}} \cdot \phi_{k j}(\mathbf{z}, \mathbf{y}),
$$

where Eq. (4.14) has been used:

$$
\begin{aligned}
& \left\langle\Lambda g_{j}(\mathbf{y}), \mathbf{g}_{1}(-\mathbf{k})\right\rangle=-\frac{k^{2} \eta}{c}\left\langle\mathscr{I}_{j}(\mathbf{y}), \mathbf{u}(\mathbf{k})\right\rangle=\frac{k^{2} \eta}{c^{*}} \frac{m}{\beta} e^{-i \mathbf{k} \cdot \boldsymbol{g}_{j}} f(\mathbf{y}) \mathbf{S}(\mathbf{k}) \Delta(\mathbf{k}), \\
& \left\langle\Lambda \mathscr{g}_{j}(\mathrm{y}), \mathscr{f}_{l}(\mathrm{z})\right\rangle=-\xi\left\langle h(\mathrm{y})\left(\mathrm{p}_{j} m^{-1}-c^{-1} \mathrm{u}\left(\mathrm{y}_{j}\right)\right), \mathscr{f}_{l}(\mathbf{z})\right\rangle-\frac{m}{c^{*}} \int \frac{d \mathbf{k}}{(2 \pi)^{3}} e^{-i \mathbf{k} \cdot y_{j}} f(\mathrm{y})\left\langle\Lambda \mathbf{g}_{\perp}(\mathbf{k}), \mathscr{f}_{l}(\mathbf{z})\right\rangle \\
& =-\beta^{-1}\left[\xi \mid \delta_{j l} \delta(\mathbf{y}-\mathbf{z}) f(\mathbf{y})-\frac{m^{2} \eta}{c^{*^{2}}} f(\mathbf{y}) f(\mathbf{z}) \frac{\partial^{2}}{\partial y_{j}^{2}} \mathbf{S}\left(\mathrm{y}_{j}-\mathbf{z}_{l}\right)\right],
\end{aligned}
$$

and

$$
\left\langle\Lambda \mathscr{J}_{j}(\mathrm{y}), \mathcal{J}_{\mathrm{y}}(-\mathbf{k}, \mathrm{z})\right\rangle=\frac{\xi}{\beta} \mathbf{S}(\mathbf{k}) \Delta(\mathbf{k}) e^{-i \mathbf{k} \cdot \mathrm{y}_{j}}[\delta(\mathrm{y}-\mathrm{z}) f(\mathrm{z})-f(\mathrm{y}) f(\mathrm{z})] .
$$

Substituting Eqs. (A8)-(A11) into (A7) yields

$$
\begin{aligned}
\mathcal{P}_{T} \Lambda \mathscr{g}_{j}(\mathrm{y})= & -\beta^{-1} \int d \mathrm{z} \sum_{k} \phi_{j k}(\mathrm{y}, \mathrm{z}) f(\mathrm{z})^{-1} \cdot\left(\frac{\partial}{\partial \mathrm{z}_{k}}-\beta \mathrm{F}^{k}(\mathrm{z})\right) h(\mathrm{z}) \\
& -\xi\left[m^{-1} \sum_{k} \int d \mathrm{z} f(\mathrm{y}) \phi_{j k}^{-1}(\mathrm{y}, \mathrm{z}) \cdot \mathscr{g}_{k}(\mathrm{z})-c^{-1} g_{u}\left(\mathrm{y}_{j}, \mathrm{y}\right)\right]+f(\mathrm{y}) \frac{m \eta}{c^{* 2}} \frac{\partial^{2}}{\partial y_{j}^{2}}\left[\frac{c^{*}}{c} \sum_{k} \int d \mathrm{z} \mathbf{s}\left(\mathrm{y}_{j}-\mathrm{z}_{k}\right) \cdot \mathscr{g}_{k}(\mathrm{z})-\mathrm{g}_{1}\left(\mathrm{y}_{j}\right)\right] .
\end{aligned}
$$

Finally, we consider 


$$
\begin{aligned}
\mathcal{O}_{T} \Lambda \mathscr{g}_{u}(\mathbf{k}, \mathbf{y})= & \int d \mathbf{z}\left\langle\Lambda \mathscr{g}_{u}(\mathbf{k}, \mathbf{y}), h(\mathbf{z})\right\rangle f(\mathbf{z})^{-1} h(\mathbf{z})+\frac{\beta}{c^{*}} \int \frac{d \mathbf{k}^{\prime}}{(2 \pi)^{3}}\left\langle\Lambda \mathscr{g}_{u}(\mathbf{k}, \mathbf{y}), \mathbf{g}_{1}\left(-\mathbf{k}^{\prime}\right)\right\rangle \cdot \mathbf{g}_{\perp}\left(\mathbf{k}^{\prime}\right) \\
& +\frac{\beta}{m} \sum_{j k} \int d \mathbf{z} d \mathbf{z}^{\prime}\left\langle\Lambda \mathscr{g}_{u}(\mathbf{k}, \mathbf{y}), \mathscr{g}_{j}(\mathbf{z})\right\rangle \cdot \phi_{j k}^{-1}\left(\mathbf{z}, \mathbf{z}^{\prime}\right) \cdot \mathscr{g}_{k}\left(\mathbf{z}^{\prime}\right)+\frac{\beta}{c} \int \frac{d \mathbf{k}^{\prime}}{(2 \pi)^{3}} d \mathbf{z}\left\langle\Lambda \mathscr{g}_{u}(\mathbf{k}, \mathbf{y}), \boldsymbol{g}_{u}\left(-\mathbf{k}^{\prime}, \mathbf{z}\right)\right\rangle f(\mathbf{z})^{-1} \mathscr{g}_{u}\left(\mathbf{k}^{\prime}, \mathbf{z}\right) .
\end{aligned}
$$

The first two terms in (A13) vanish. The second two are given by

$$
\begin{aligned}
& \left\langle\Lambda \mathcal{J}_{u}(\mathbf{k}, \mathbf{y}), \mathscr{Z}_{j}(\mathbf{z})\right\rangle=\frac{\xi}{\beta} e^{i \mathbf{k} \cdot \mathbf{z}_{j}} \mathbf{S}(\mathbf{k}) \Delta(\mathbf{k})[\delta(\mathbf{y}-\mathbf{z}) f(\mathbf{y})-f(\mathbf{y}) f(\mathbf{z})], \\
& \left\langle\Lambda \mathcal{J}_{u}(\mathbf{k}, \mathbf{y}), \mathscr{I}_{u}\left(-\mathbf{k}^{\prime}, \mathbf{z}\right)\right\rangle=-\frac{k^{2} \eta}{c}\left\langle\mathcal{I}_{u}(\mathbf{k}, \mathbf{y}), \mathscr{I}_{u}\left(-\mathbf{k}^{\prime}, \mathbf{z}\right)\right\rangle-\frac{\xi}{c} \sum_{j} \mathbf{s}(\mathbf{k}) \Delta(\mathbf{k}) \\
& \times \int d \mathbf{w} e^{i \mathbf{k} \cdot \mathbf{w}_{f}}(\delta(\mathbf{w}-\mathbf{y})-f(\mathbf{y})) \int \frac{d \mathbf{q}}{(2 \pi)^{3}} e^{-i \mathbf{q} \cdot \mathbf{w}_{j}}\left\langle\mathcal{I}_{u}(\mathbf{q}, \mathbf{w}), \mathscr{g}_{u}\left(-\mathbf{k}^{\prime}, \mathbf{z}\right)\right\rangle .
\end{aligned}
$$

Therefore

$$
\mathcal{P}_{T} \Lambda \mathcal{J}_{u}(\mathbf{k}, \mathbf{y})=-\frac{k^{2} \eta}{c} \mathscr{I}_{u}(\mathbf{k}, \mathbf{y})+\xi \sum_{j} \mathbf{s}(\mathbf{k}) \Delta(\mathbf{k}) \int d \mathbf{z} e^{i \mathbf{k} \cdot \mathbf{z}_{j}}[\delta(\mathbf{y}-\mathbf{z})-f(\mathbf{y})]\left\{m^{-1} \sum_{k} \int d \mathbf{z}^{\prime} \phi_{j k}^{-1}\left(\mathbf{z}, \mathbf{z}^{\prime}\right) f(\mathbf{z}) \cdot \mathcal{Z}_{k}(\mathbf{z})-\frac{1}{c} \mathcal{J}_{u}\left(\mathbf{z}_{j}, \mathbf{z}\right)\right\} .
$$

\section{APPENDIX B: $\left\langle\mathcal{S}_{i}(y, t), T\right\rangle$}

Equation (4.38) may be rewritten as

$$
\begin{aligned}
& \xi\left[m^{-1} \sum_{k} \int d \mathbf{z} f(\mathbf{y}) \phi_{j k}^{-1}(\mathbf{y}, \mathbf{z}) \cdot\left\langle\mathscr{g}_{k}(\mathbf{z}, t), \mathrm{T}\right\rangle-c^{-1}\left\langle\mathscr{I}_{u}\left(\mathbf{y}_{j}, \mathbf{y}, t\right), \mathrm{T}\right\rangle\right] \\
& =-\beta^{-1}\left(\frac{\partial}{\partial \mathbf{y}_{j}}-\beta \mathbf{F}^{j}(\mathbf{y})\right)\langle h(\mathbf{y}, t), \mathbf{T}\rangle-\frac{m}{c^{*}} f(\mathbf{y}) \sum_{k} \int d \mathbf{z} \mathbf{S}\left(\mathbf{y}_{j}-\mathbf{z}_{k}\right) \cdot \mathbf{F}^{k}(\mathbf{z})\langle h(\mathbf{z}, t), \mathbf{T}\rangle+\frac{m}{c^{*}} f(\mathbf{y}) \int \frac{d \mathbf{q}}{(2 \pi)^{3}} e^{-i \mathbf{q}^{*} \mathbf{y}_{j}} \\
& \times\left\{-\frac{q^{2} \eta}{c} \mathbf{S}(\mathbf{q}) \Delta(\mathbf{q}) \sum_{k} \int d \mathbf{z} e^{i \boldsymbol{q} \cdot \mathbf{z}_{k}}\left\langle\boldsymbol{g}_{k}(\mathbf{z}, t), \mathbf{T}\right\rangle+\frac{q^{2} \eta}{c}\left\langle\mathbf{g}_{\perp}(\mathbf{q}, t), \mathbf{T}\right\rangle\right\} \\
& =-\beta^{-1}\left(\frac{\partial}{\partial \mathbf{y}_{j}}-\beta \mathbf{F}^{j}(\mathbf{y})\right)\langle h(\mathbf{y}, t), \mathrm{T}\rangle-\frac{m}{c^{*}} f(\mathbf{y}) \frac{\partial}{\partial t}\left\langle\mathbf{g}_{1}\left(\mathbf{y}_{j}, t\right), \mathrm{T}\right\rangle,
\end{aligned}
$$

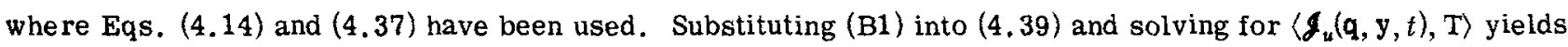
$\left\langle\mathcal{g}_{u}(\mathbf{q}, \mathbf{y}, t), \mathbf{T}\right\rangle=-\frac{c}{\eta} \sum_{j} q^{-2} \mathbf{S}(\mathbf{q}) \Delta(\mathbf{q}) \int d \mathbf{z} e^{i \mathbf{s} \cdot \mathbf{z}_{j}(\delta(\mathbf{y}-\mathbf{z})-f(\mathbf{y}))}\left\{\beta^{-1}\left(\frac{\partial}{\partial \mathbf{z}_{j}}-\beta \mathbf{F}^{j}(\mathbf{z})\right)\langle h(\mathbf{z}, t), \mathbf{T}\rangle-\frac{m}{c^{*}} f(\mathbf{z}) \frac{\partial}{\partial t}\left\langle\mathbf{g}_{1}\left(\mathbf{z}_{j}, t\right), \mathbf{T}\right\rangle\right\}$.

In view of Eqs. (B1) and (B2) we write

$$
\begin{aligned}
& \left\langle\mathcal{J}_{j}(\mathbf{y}, t), \mathrm{T}\right\rangle=\sum_{k} \int d \mathbf{z} \phi_{j k}(\mathbf{y}, \mathbf{z}) f(\mathbf{z})^{-1} \cdot\left\{\sum_{l} \int d \mathbf{w} f(\mathbf{z}) \phi_{k l}^{-1}(\mathbf{z}, \mathbf{w})\left\langle\mathscr{d}_{l}(\mathbf{w}, t), \mathrm{T}\right\rangle-\frac{m}{c}\left\langle\mathcal{g}_{u}\left(\mathbf{z}_{k}, \mathbf{z}, t\right), \mathrm{T}\right\rangle\right\} \\
& +\sum_{k} \frac{m}{c} \int d \mathbf{z}_{\phi_{j k}}(\mathbf{y}, \mathbf{z}) \cdot\left\langle\mathscr{J}_{u}\left(\mathbf{z}_{k}, \mathbf{z}, t\right), \mathrm{T}\right\rangle f(\mathbf{z})^{-1} \\
& =-m \sum_{k} \int d \mathbf{z} \phi_{j k}(\mathbf{y}, \mathbf{z}) f(\mathbf{z})^{-1}\left\{\left(\xi \beta^{-1}\right)\left(\frac{\partial}{\partial \mathbf{z}_{k}}-\beta \mathrm{F}^{k}(\mathbf{z})\right)\langle h(\mathbf{z}, t), \mathbf{T}\rangle+\frac{m}{\xi c} f(\mathbf{z}) \frac{\partial}{\partial t}\left\langle\mathbf{g}_{1}\left(\mathbf{z}_{k}, t\right), \mathbf{T}\right\rangle\right\} \\
& -\sum_{k l} \frac{m}{c} \int d \mathbf{z} d \mathbf{w} \phi_{j k}(\mathbf{y}, \mathbf{z}) f(\mathbf{z})^{-1} \cdot \mathbf{T}_{l}^{k l}(\mathbf{z}, \mathbf{w}) \cdot[\delta(\mathbf{z}-\mathbf{w})-f(\mathbf{z})]\left\{\beta^{-1}\left(\frac{\partial}{\partial \mathbf{w}_{l}}-\beta \mathbf{F}^{l}(\mathbf{w})\right)\langle h(\mathbf{w}, t), \mathbf{T}\rangle+\frac{m}{c} f(\mathbf{w}) \frac{\partial}{\partial t}\left\langle\mathbf{g}_{1}\left(\mathbf{w}_{l}, t\right), \mathbf{T}\right\rangle\right\},
\end{aligned}
$$

where

$$
\mathbf{T}_{i}^{l k}(\mathbf{z}, \mathrm{w}) \equiv \frac{1}{\eta} \int \frac{d \mathbf{k}}{(2 \pi)^{3}} \exp \left[-i \mathbf{k} \cdot\left(\mathbf{z}_{l}-\mathrm{w}_{k}\right)\right] k^{-2} \Delta(\mathbf{k}) \mathbf{s}(\mathbf{k})
$$

In Eq. (B3) the term $\sim(\theta / \partial t)\left\langle\mathbf{g}_{1}(\mathbf{k}, t), \mathrm{T}\right\rangle$ will be neglected due to the restriction $t \gg m / \xi$. Here we also assume that $\xi / m \sim \eta l$. Therefore, Eq. (B3) becomes

$$
\left\langle\mathcal{Y}_{j}(\mathbf{y}, t), \mathrm{T}\right\rangle \simeq-m \sum_{k} \int d \mathbf{z} \mathbf{D}^{j k}(\mathbf{y}, \mathbf{z}) \cdot\left(\frac{\theta}{\partial \mathbf{z}_{k}}-\beta \mathrm{F}^{k}(\mathbf{z})\right)\langle h(\mathbf{z}, t), \mathbf{T}\rangle,
$$

with

$$
\mathbf{D}^{j k}(\mathrm{y}, \mathrm{z}) \equiv \int d \mathrm{w} \sum_{l} \phi_{j l}(\mathrm{y}, \mathrm{w}) f(\mathrm{w})^{-1}\left\{\delta_{l k} \delta(\mathrm{w}-\mathrm{z})(\beta \xi)^{-1}+\mathbf{T}_{l}^{l k}(\mathrm{w}, \mathbf{z})(\delta(\mathrm{w}-\mathrm{z})-f(\mathrm{w}))\right\}
$$


${ }^{1} \mathrm{H}$. Yamakawa, Modern Theory of Polymer Solutions (Harper and Row, New York, 1971).

${ }^{2}$ P. J. Flory, Principles of Polymer Chemistry (Cornell U. P., Ithaca, 1953).

${ }^{3} \mathrm{~J}$. D. Ferry, Viscoelastic Properties of Polymers (Wiley, New York, 1970), 2nd ed.

${ }^{4}$ J. G. Kirkwood and J. Riseman, J. Chem. Phys. 16, 549 (1948); J. G. Kirkwood and J. Riseman, in Rheology, Theory and Applications, edited by F. R. Eirich (Academic, New York, 1956), p. 495.

${ }^{5}$ B. H. Zimm, J. Chem. Phys. 24, 269 (1956).

${ }^{6}$ S. F. Edwards and K. F. Freed, J. Chem. Phys, 61, 1189 (1974).

${ }^{7}$ K. F. Freed and S. F. Edwards, J. Chem. Phys. 61, 3626 (1974).

${ }^{8}$ P. E. Rouse, J. Chem. Phys. 21, 1271 (1953).

${ }^{9}$ H. Yamakawa, G. Tanaka, and W. H. Stockmayer, J. Chem. Phys. 61, 4535 (1974).

${ }^{10}$ W. H. Stockmayer, G. Wilemski, H. Yamakawa, and G. Tanaka, J. Chem. Phys. 63, 1039 (1975).

${ }^{11} \mathrm{C}$. F. Curtiss, R. B. Bird, and $\mathrm{O}$. Hassager, Am, Chem. Soc., Div. Polym. Chem. 15, 1 (1974).

${ }^{12}$ R. Kapral, D. Ng, and S. G. Whittington, J. Chem. Phys. 64, 539 (1976).

${ }^{13}$ R. S. Adler, Doctoral Dissertation, Dept. Nucl. Eng., Univ. of Michigan (1977).

${ }^{14}$ W. H. Stockmayer, W. Gobush, Y. Chibahisa, and D. K. Carpenter, Discuss. Faraday Soc. 49, 182 (1970).

${ }^{15}$ L. P. Kadanoff and P. C. Martin, Ann. Phys. 24, 419 (1963).
${ }^{16} \mathrm{M}$. Doi and K. Okano, Polymer J. 5, 216 (1973).

${ }^{17}$ K. Kawasaki, Ann. Phys. 61, 1 (1970).

${ }^{18}$ M. Fixman, J. Chem. Phys. 36, 310 (1962).

${ }^{19}$ A. Z. Akcasu and H. Gürol, J. Polym. Sci., Phys. Ed. 14, 1 (1976).

${ }^{20}$ H. Mori and H. Fujisaka, Prog. Theor. Phys. 49, 1516 (1973).

${ }^{21} \mathrm{R}$. Zwanzig, J. Chem. Phys. 33, 423 (1960).

${ }^{22}$ The notation to be used is discussed by J. Rzewuski, in Field Theory (PWN Polish Scientific Publ., Warsaw, 1969), Vol. 2.

${ }^{23}$ H. Mori, Prog. Theor. Phys. 51, 109 (1974).

${ }^{24}$ In Mori's expansion higher order terms appear as powers of $[\delta / \delta \mathbf{a} \cdot Q \dot{\mathbf{A}}]$.

${ }^{25}$ M. Bixon, J. Chem. Phys. 58, 1459 (1973).

${ }^{26}$ R. Zwanzig, J. Chem. Phys. 60, 2717 (1974).

${ }^{27}$ K. F. Freed, S. F. Edwards, and M. Warner, J. Chem. Phys. 64, 5132 (1976).

${ }^{28}$ Coarse-graining in this manner is discussed by $\mathrm{H}$. Mori, Prog. Theor. Phys, 49, 1516 (1973).

${ }^{29}$ J. Lebowitz and E. Rubin, Phys. Rev. 131, 2381 (1963).

${ }^{30} \mathrm{Zwanzig}$ has also suggested this type of expansion in connection to the fluctuation renormalization of transport coefficients, in Proceedings of the Sixth IUPAP Conference on Statistical Mechanics, edited by S. A. Rice, K. F. Freed, and J. C. Light (University of Chicago Press, Chicago, 1972), p. 241.

${ }^{31}$ H. Mori, Prog. Theor. Phys. 33, 423 (1965).

${ }^{32} \Delta$ is taken as the smallest characteristic length entering the problem.

${ }^{33}$ This limit corresponds to neglecting all terms connecting two or more chains. 